\title{
Bioanalysis
}

\section{White Paper On Recent Issues in Bioanalysis: FDA BMV Guidance, ICH M10 BMV Guideline and Regulatory Inputs (Part 2 - Recommendations on 2018 FDA BMV Guidance, 2019 ICH M10 BMV Draft Guideline and Regulatory Agencies' Input on Bioanalysis, Biomarkers and Immunogenicity)}

Brian Booth ${ }^{\ddagger}, \#, 1$, Lauren Stevenson $\$, \S, 13$, Renuka Pillutla ${ }^{\ddagger 10}$, Michael Buonarati ${ }^{\ddagger} \S, 3$, Chris Beaver $\ddagger, 2$, Daniela Fraier $\ddagger, 4$, Fabio Garofolo*, $, \S, 5$, Sam Haidar $\ddagger, 1$, Rafiq Islam ${ }^{\ddagger} \S, 6$, Christopher James ${ }^{\ddagger, 7}$, John Kadavil ${ }^{\ddagger} \#, 1$, Olga Kavetska ${ }^{\ddagger}, 8$, Fumin Li $^{\ddagger, 9}$, Christina Satterwhite ${ }^{\ddagger}, \S, 11$, Natasha Savoie ${ }^{\ddagger}, \S, 12$, Sriram Subramaniam ${ }^{\ddagger}, \S, \#, 1$, Nilufer Tampal ${ }^{\ddagger} \S, \#, 1$, Theingi Thway $\$, \S, \#, 1$, Eric Woolf $\$, 14$, Olivier Le Blaye ${ }^{\S, \#, 22}$, Matthew Andisik $^{\S, 15}$, Chad Briscoe ${ }^{\S, 16}$, Stephanie Cape ${ }^{\S, 17}$, Arindam Dasgupta ${ }^{\S, \#, 1}$, Sally Fischer $^{\S, 18}$, Sam Haidar $\$, \#, 1$, Roger Hayes ${ }^{\S, 19}$, John Kamerud ${ }^{\S 21}$, Gustavo Mendes Lima Santos $\$, \#, 23$, Corey Nehls ${ }^{\S, 24}$, Catherine Soo ${ }^{\S, \#, 25}$, Stephen Vinter ${ }^{\S, \#, 26}$, Emma Whale ${ }^{\S \#, 26}$, Keyang $\mathrm{Xu}^{\S, 18}$, Seongeun (Julia) Cho ${ }^{\#, 1}$, Anna Edmison ${ }^{\#, 25}$, Sean Kassim,1, Thais Correa Rocha\#,23, Jan Welink",27, Shashi Amur ${ }^{\mathbb{1}, 1}$, Abbas Bandukwala $\mathbb{1}, 1$, Elana Cherry $\mathbb{\mathbb { 1 } 2 5}$, Shirley Hopper ${ }^{\mathbb{1}, 28}$, Akiko Ishii-Watabe $\mathbb{I}^{\mathbb{2} 29}$, Susan Kirshner $\mathbb{I}^{\mathbb{1}}$, Kevin Maher $\mathbb{\mathbb { I }}^{1}$, Joao Pedras-Vasconcelos $\mathbb{1}^{\mathbb{1}}$, Yoshiro Saito ${ }^{\mathbb{I}, 29}$, Therese Solstad Saunders ${ }^{\mathbb{1}, 30}$, Venke Skibeli ${ }^{\mathbb{I}, 30}$, Daniela Verthelyi ${ }^{\mathbb{1}, 1}$, Yow-Ming Wang ${ }^{\mathbb{I}, 1}$ \& Haoheng Yan $\mathbb{1} 1$

\footnotetext{
${ }^{1}$ US FDA, Silver Spring, MD, USA

${ }^{2}$ Syneos Health, Princeton, NJ, USA

${ }^{3}$ Intertek, San Diego, CA, USA

${ }^{4}$ F. Hoffmann-La Roche Ltd, Basel, Switzerland

${ }^{5}$ Angelini Pharma, Rome, Italy

${ }^{6}$ Celerion, Lincoln, NE, USA

${ }^{7}$ Amgen Research, Thousand Oaks, CA, USA

${ }^{8}$ Pfizer, Groton, CT, USA

${ }^{9} \mathrm{PPD}$, Middleton, WI, USA

${ }^{10}$ Bristol-Myers Squibb, Princeton, NJ, USA

${ }^{11}$ Charles River, Reno, NV, USA

${ }^{12}$ CFABS, Montreal, QC, Canada

${ }^{13}$ Biogen, Cambridge, MA, USA

${ }^{14}$ Merck Research Labs, West Point, PA, USA

${ }^{15}$ Regeneron Pharmaceuticals, Tarrytown, NY, USA
}

\author{
${ }^{16}$ PRA Health Sciences, Lenexa, KS, USA \\ ${ }^{17}$ Covance, Madison, WI, USA \\ ${ }^{18}$ Genentech, South San Francisco, CA, USA \\ ${ }^{19}$ ICON, Whitesboro, NY, USA \\ ${ }^{20} \mathrm{GlaxoSmithKline,} \mathrm{King} \mathrm{of} \mathrm{Prussia,} \mathrm{PA,} \mathrm{USA}$ \\ ${ }^{21}$ Pfizer, Andover, MA, USA \\ ${ }^{22}$ France ANSM, Saint Denis, France \\ ${ }^{23}$ Brazil ANVISA, Brasilia, Brazil \\ ${ }^{24} \mathrm{PPD}$, Richmond, VA, USA \\ ${ }^{25}$ Health Canada, Ottawa, ON, Canada \\ ${ }^{26}$ UK MHRA, London, UK \\ ${ }^{27} \mathrm{EMA}$, London, UK \\ ${ }^{28}$ UK MHRA-NIBSC, London, UK \\ ${ }^{29}$ Japan MHLW-NIHS, Tokyo, Japan \\ ${ }^{30}$ Norwegian Medicines Agency (NoMA), Oslo, Norway
}

The $201913^{\text {th }}$ Workshop on Recent Issues in Bioanalysis (WRIB) took place in New Orleans, LA on April 1-5, 2019 with an attendance of over 1000 representatives from pharmaceutical/biopharmaceutical companies, biotechnology companies, contract research organizations and regulatory agencies worldwide. WRIB was once again a 5-day, week-long event - a full immersion week of bioanalysis, biomarkers, immunogenicity and gene therapy. As usual, it was specifically designed to facilitate sharing, reviewing, discussing and agreeing on approaches to address the most current issues of interest including both small- and large-molecule bioanalysis involving LCMS, hybrid LBA/LCMS, LBA cell-based/flow cytometry assays and qPCR approaches. This 2019 White Paper en-

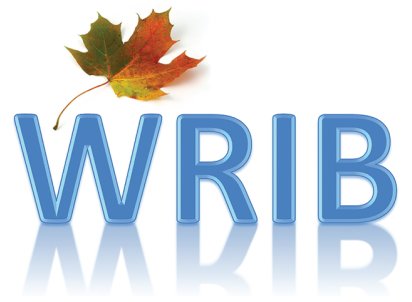

$¥$ Section 1 - Implementation of 2018 FDA BMV guidance (authors are presented in alphabetical order of their last name, with the exception of the first four who were session chairs, working dinner facilitators and/or major contributors).

§ Section 2 - Feedback on ICH M10 BMV draft guideline (authors are presented in alphabetical order of their last name, with the exception of the first three who were major contributors and/or notetakers). Note: Brian Booth (US FDA), Jan Welink (EMA), Anna Edmison (Health Canada), Akiko Ishii-Watabe (MHLW-NIHS), Yoshiro Saito (MHLW) and Thais Correa Rocha (ANVISA) from the ICH M10 EWG helped, organized, coordinated and participated in the ICH M10 discussions and activities throughout WRIB. Their purpose was to attend WRIB, provide clarity on ICH M10 and seek public input on the draft document. The ICH M10 EWG neither endorses nor refutes any of the below recommendations, nor are they authors of these remarks due to conflict of interest.

\# Section 3 - Input from regulatory agencies on bioanalysis and BMV (authors are presented in alphabetical order of their last name)

I Section 4 - Input from regulatory agencies on immunogenicity and biomarkers (authors are presented in alphabetical order of their last name). Disclaimer: The views expressed in this article are those of the authors and do not reflect official policy of the US FDA, Europe EMA, France ANSM, UK MHRA, Brazil ANVISA, Health Canada, Japan MHLW, and Norway NoMA. No official endorsement by the FDA, EMA, ANSM, Health Canada, MHRA, ANVISA, MHLW or NoMA is intended or should be inferred.

*Author for correspondence: fabiogarofolo@hotmail.com 
compasses recommendations emerging from the extensive discussions held during the workshop, and is aimed to provide the bioanalytical community with key information and practical solutions on topics and issues addressed, in an effort to enable advances in scientific excellence, improved quality and better regulatory compliance. Due to its length, the 2019 edition of this comprehensive White Paper has been divided into three parts for editorial reasons. This publication (Part 2) covers the recommendations on the 2018 FDA BMV guidance, 2019 ICH M10 BMV draft guideline and regulatory agencies' input on bioanalysis, biomarkers, immunogenicity and gene therapy. Part 1 (Innovation in Small Molecules and Oligonucleotides and Mass Spectrometry Method Development Strategies for Large-Molecule Bioanalysis) and Part 3 (New Insights in Biomarker Assay Validation, Current and Effective Strategies for Critical Reagent Management, Flow Cytometry Validation in Drug Discovery and Development and CLSI H62, Interpretation of the 2019 FDA Immunogenicity Guidance and Gene Therapy Bioanalytical Challenges) are published in volume 10 of Bioanalysis, issues 22 and 24 (2019), respectively.

First draft submitted: 24 October 2019; Accepted for publication: 30 October 2019; Published online: 13 December 2019

\section{Key terms}

Biomarker: A defined characteristic that is measured as an indicator of normal biological processes, pathogenic processes, or responses to an exposure or intervention, including therapeutic interventions. Molecular, histologic, radiographic, or physiologic characteristics are types of biomarkers. Categories of biomarkers include: susceptibility/risk biomarker, diagnostic biomarker, monitoring biomarker, prognostic biomarker, predictive biomarker, pharmacodynamic/response biomarker and safety biomarker [60].

Biomarker qualification: A conclusion based on a formal regulatory process, that within the stated context of use, a medical product development tool can be relied upon to have a specific interpretation and application in medical product development and regulatory review [60].

Immunogenicity: The ability of a substance, including a biotherapeutic substance, to provoke an immune response in vivo which results in an induction of anti-drug antibodies and immune cell activation, etc. For the purposes of this article, anti-drug antibody assays are equivalent to immunogenicity assays.

Cut point: The minimum level of response in the assay that defines the sample as positive for anti-drug antibody.

Context of use: A statement that fully and clearly describes the way the medical product development tool is to be used and the medical product development-related purpose of the use [60].

\section{Index}

- Introduction

- Section 1 - Implementation of 2018 FDA BMV guidance

- Discussion topics

- Discussions, consensus and conclusions

- Recommendations

- Section 2 - Feedback on ICH M10 BMV draft guidelines

- Discussion topics

- Discussions, consensus and conclusions

- Recommendations

- Section 3 - Input from regulatory agencies on bioanalysis \& BMV

- Regulatory inputs

- Q \& A

- Section 4 - Input from regulatory agencies on immunogenicity \& biomarkers

- Regulatory inputs

- $\mathrm{Q} \& \mathrm{~A}$

- References 


\section{Acronyms}

ADA: Anti-drug antibody

BA: Bioavailability

BAV: $\quad$ Biomarker assay validation

BE: Bioequivalence

BLA: Biologics license application

BMV: Bioanalytical method validation

CAPA: Corrective and preventive actions

CDx: Companion diagnostics

CMC: chemistry, manufacturing and control

COU: Context of use

CRO: Contract Research Organization

DBS: Dried blood spots

GCP: Good Clinical Practice

HC: $\quad$ Health Canada

HRMS: High-resolution mass spectrometry

IA: Immunoaffinity

IND: Investigational new drug

ISR: Incurred sample reanalysis

IVDR: In vitro diagnostic medical device

LBA: Ligand-binding assay

LCMS: Liquid chromatography mass spectrometry

LLOQ: Lower limit of quantitation

LM: $\quad$ Large molecule

LTS: Long-term stability

mAb: Monoclonal antibody

MOA: Mechanism of action

MRD: Minimum required dilution

MS: $\quad$ Mass spectrometry

Nab: Neutralizing antibody

PC: Positive control

PD: Pharmacodynamics

PK: $\quad$ Pharmacokinetics

QC: Quality control

QP: Qualification plan

S/N: Signal-to-noise

SIL-IS: $\quad$ Stable isotope label internal standard

ULOQ: Upper limit of quantitation

WRIB: $\quad$ Workshop on Recent Issues in Bioanalysis

\section{Introduction}

The $13^{\text {th }}$ Workshop on Recent Issues in Bioanalysis (13 ${ }^{\text {th }}$ WRIB) was held in New Orleans, LA on 1-5 April 2019 with an attendance of over 1000 representatives from pharmaceutical/biopharmaceutical companies, biotechnology companies, contract research organizations, and regulatory agencies worldwide. The workshop included 3 sequential main workshop days, six additional full-day training sessions that together spanned an entire week to allow exhaustive and thorough coverage of all major issues in bioanalysis, biomarkers, immunogenicity and gene therapy. 
As in previous years, this year's WRIB continued to gather a wide diversity of international industry opinion leaders and regulatory authority experts working on both small and large molecules to facilitate sharing of data, ideas and fostering discussions focused on improving quality, increasing regulatory compliance and achieving scientific excellence on bioanalytical issues.

The active contributing chairs included Dr Christine Fandozzi (Merck), Dr Christopher Evans (GlaxoSmithKline), Dr Brian Booth (US FDA), Dr Renuka Pillutla (Bristol-Myers Squibb), Dr Fabio Garofolo (Angelini Pharma), Dr Becky Schweighardt (BioMarin), Dr Meina Liang (AstraZeneca) and Dr Lauren Stevenson (Biogen).

The participation of regulatory agency representatives continued to grow at WRIB [1-21] and included the below representatives:

- Regulated bioanalysis: Dr Sean Kassim (US FDA), Dr Sam Haidar (US FDA), Dr Seongeun (Julia) Cho (US FDA), Dr John Kadavil (US FDA), Dr Arindam Dasgupta (US FDA), Dr Brian Booth (US FDA), Dr Sriram Subramaniam (US FDA), Dr Theingi Thway (US FDA), Dr Nilufer Tampal (US FDA), Dr Jan Welink (EU EMA), Dr Olivier Le Blaye (France ANSM), Mr Stephen Vinter (UK MHRA), Ms Emma Whale (UK MHRA), Dr Anna Edmison (Health Canada), Dr Catherine Soo (Health Canada), Mr Gustavo Mendes Lima Santos (Brazil ANVISA), Ms Thais Correa Rocha (Brazil ANVISA)

- Biomarkers: Dr Yow-Ming Wang (US FDA), Dr Abbas Bandukwala (US FDA), Dr Kevin Maher (US FDA), Dr Shashi Amur (US FDA), Dr Shirley Hopper (UK MHRA), Dr Yoshiro Saito (Japan MHLW-NIHS)

- Immunogenicity: Dr João Pedras-Vasconcelos (US FDA), Dr Haoheng Yan (US FDA), Dr Susan Kirshner (US FDA) - remote, Dr Daniela Verthelyi (US FDA) - remote, Dr Elana Cherry (Health Canada), Dr Akiko Ishii-Watabe (Japan MHLW-NIHS), Dr Venke Skibeli (Norway NoMA), Dr Therese Solstad Saunders (Norway NoMA)

- Gene therapy: Dr Nirjal Bhattarai (US FDA), Dr Heba Degheidy (US FDA)

The $13^{\text {th }}$ WRIB was designed to cover a wide range of topics in bioanalysis, biomarkers, immunogenicity and a special full-day session dedicated to gene therapy bioanalytical challenges. Moreover, the $13^{\text {th }}$ WRIB included lectures from both industry experts and regulatory representatives and daily working dinners where key topics were discussed and debated. These efforts culminated in open panel discussions amongst the presenters, regulators and attendees to reach consensus on items presented in this White Paper.

While the $13^{\text {th }}$ WRIB continued its traditional emphasis on method development challenges and novel solutions in bioanalysis, it also included an in-depth focus on the recently released ICH M10 draft guideline [22] for bioanalytical method validation. Three full sessions, two working dinners and three open forums were dedicated to cover the hot topics of the ICH M10 draft guideline and to actively interact with the regulators' expert panel and work together as a global bioanalytical community with the goal to provide official comments on the ICH M10 BMV draft guideline. Harmonized topics among US FDA, EU EMA, Health Canada, Japan MHLW and Brazil ANVISA regulations, unresolved issues and ongoing industry/regulator discussions were thoroughly evaluated to support the regulatory requirements of the ICH M10 draft guideline which, when finalized, will supersede the regional guidance of the participating health authorities.

The three sessions and open forums on ICH M10 activities at WRIB were organized and coordinated by Dr Brian Booth (US FDA, ICH M10 EWG Regulatory Chair), Dr Jan Welink (EU EMA), Dr Anna Edmison (Health Canada), Dr Akiko Ishii-Watabe (MHLW, ICH M10 EWG Rapporteur), Dr Yoshiro Saito (MHLW), and Ms Thais Correa Rocha (Brazil ANVISA) and with input and active participation from numerous industry/regulator opinion leaders.

A total of 48 recent issues ('hot topics') were addressed and distilled into a series of relevant recommendations. Presented in the current White Paper is the background on each issue, exchanges, consensus and resulting recommendations on these 48 topics.

Due to its length, the 2019 edition of this comprehensive White Paper has been divided into three parts for editorial reasons. This publication covers Part 2 conclusions.

Part 1 - Bioanalysis Volume 11, Issue 22 (November 2019)

Innovation in small molecules and oligonucleotides:

- Novel therapeutic modalities (2 topics); 
- Innovation in small molecules (3 topics);

- Small-molecule biomarkers by LCMS (1 topic);

- Oligonucleotides (1 topic);

Mass spectrometric method development strategies for large-molecule bioanalysis:

- Innovation in hybrid LBA/LCMS assays (5 topics);

- Biomarker assays (3 topics);

Part 2 - Bioanalysis Volume 11, Issue 23 (December 2019)

Implementation of 2018 FDA BMV guidance (10 topics)

Industry/regulators' feedback on ICH M10 BMV draft guidelines (14 topics)

Input from regulatory agencies on bioanalysis \& BMV

Input from regulatory agencies on immunogenicity \& biomarkers

Part 3 - Bioanalysis Volume 11, Issue 24 (December 2019)

New insights in biomarker assay validation (BAV):

- Fit for purpose and context of use (2 topics);

- Free assays (1 topic);

- BAV guidelines (2 topics);

Current \& effective strategies for critical reagent management:

- Characterization and stability (3 topics);

- Life cycle management (1 topic);

- Flow cytometry (1 topic);

- Challenges and approaches (1 topic);

Flow cytometry validation in drug discovery $\&$ development $\&$ CLSI H62:

- Regulatory expectations and validation (2 topics);

- Challenges and approaches (3 topics);

- Data analysis (2 topics);

Interpretation of the 2019 FDA immunogenicity guidance:

- Drug tolerance (1 topic);

- Critical reagent and positive control characterization (1 topic);

- LCM and clinical relevance of ADA (1 topic);

- Challenges and approaches (3 topics);

- Cut points and the FDA immunogenicity guidance (1 topic);

Gene therapy bioanalytical challenges:

- Approaches to gene therapy bioanalysis (4 topics);

- Vaccines (1 topic);

- Challenges (2 topics);

- Immunogenicity (1 topic).

\section{Section 1 - Implementation of 2018 FDA BMV guidance}

Brian Booth ${ }^{1}$, Lauren Stevenson ${ }^{13}$, Renuka Pillutla ${ }^{10}$, Michael Buonarati $^{3}$, Chris Beaver ${ }^{2}$, Daniela Fraier 4 , Fabio Garofolo ${ }^{5}$, Sam Haidar ${ }^{1}$, Rafiq Islam ${ }^{6}$, Christopher James ${ }^{7}$, John Kadavil ${ }^{1}$, Olga Kavetska ${ }^{8}$, Fumin Li $^{9}$, Christina Satterwhite ${ }^{11}$, Natasha Savoie ${ }^{12}$, Sriram Subramaniam ${ }^{1}$, Nilufer Tampal ${ }^{1}$, Theingi Thway ${ }^{1}$, Eric Woolf ${ }^{14}$

Authors are presented in alphabetical order of their last name, with the exception of the first four who were session chairs, working dinner facilitators or major contributors. Author affiliations can be found at the beginning of the article. 
The most recent FDA guidance on bioanalytical method validation was released in May 2018 [23]. It comes 5 years after the draft revised guidance that was issued in 2013 [24], and 17 years after the prior final guidance was issued in 2001 [25].

Overall, the well-established evaluations detailed in the chromatographic assays section remained mainly unchanged except for:

- Validation acceptance criteria for dilution and carryover;

- Clarification of the number of QC levels and replicates;

- No acceptance criteria for QCs in accuracy and precision runs;

- QCs should cover the study sample concentration range during in-study analysis;

- Lack of interference at the LLOQ should be evaluated in each run;

- Additional acceptance criteria for distinct batches within a run;

- IS response and drift which should be monitored.

Regarding LBA, additional clarity was added to:

- Accuracy and precision runs;

- The topic of anchor points;

- Emphasis is placed on:

- The inclusion of proper controls in each parameter with clear definitions for each control;

- Consistency in standard calibrator preparations used in every run.

The most significant changes made to the final guidance are:

- The incurred sample reanalysis (ISR) section;

- The addition of a section addressing:

- Endogenous compounds;

- Biomarkers;

- Diagnostic kits;

- Bridging data and dried blood spots (DBS).

The recommendations for documentation are largely unchanged but include clarifications and small changes in emphasis:

- Records must still be contemporaneous and re-constructible to ensure data integrity;

- Emphasis is placed on including all information around rejected runs and investigations;

- For chromatographic methods this includes information on reanalyzed data, including investigations, reinjections and reintegrations.

- More details are now requested to limit the need for onsite data review.

There are three typographical errors in the guidance document:

- Table 1 selectivity (CCs): "spiked samples should be $\pm 20 \%$ LLOQ" should read, “ blank samples should be no more than 20\% of the LLOQ response level";

- Tables 1 specificity (LBAs): "potential interfering materials should be added to calibration curves in buffer" should read, "potential interfering materials should be added to QCs";

- Table 1 calibration curve (LBAs): "anchor points should not be included in the curve fit" should read, "anchor points should be used to fit the curve but should not be used to count the minimum number of acceptable standard levels."

Discussion topics \& consolidated questions collected from the global bioanalytical community The following paragraphs report the consolidated questions collected from the global bioanalytical community. Ten discussion topics were extracted from these questions and considered as the most relevant 'hot topics' for the 2018 FDA BMV guidance. They were reviewed by internationally recognized opinion leaders before being submitted 
for discussion during the $13^{\text {th }}$ WRIB. The background on each issue, discussions, consensus and conclusions are in the next section and a summary of the key recommendations is provided in the final section of this manuscript.

\section{Common topics (chromatographic assays \& LBA) \\ Synopsis of method development}

What level of detail is required in the synopsis of method development to meet FDA requirements? Is there any guidance from FDA on where to place this information in the validation report? How has it been implemented in the industry? Where do you report 'unique aspects' if not part of method development? Is a method development report required for FDA audits/inspections?

\section{Stability of ultra-high concentration samples}

Must > ULOQ QCs used for stability evaluations bracket study sample concentrations or can they reside near to where the majority of > ULOQ study sample concentrations are observed? If a stability interval passes for low and high QCs, but fails at the > ULOQ QC, can a single re-analysis stability assessment be used to establish stability if acceptance criteria are subsequently met for the > ULOQ QC stability samples (e.g., previous failure likely due to sample dilution error)? If low and high QC stability is established, but not for > ULOQ QCs, can stability be considered established for study samples within the calibration range of the assay? If stability cannot be established using > ULOQ stability QC samples, can the assay be re-validated at a higher calibration range and stability re-assessed to override the > ULOQ stability results? Is there alignment that low concentration samples are most sensitive indicators of stability for most large molecule and nucleic acid therapeutics? What are the specific special cases where evaluation of high concentration samples is supported by scientific rationale?

\section{Freshly prepared calibrators \& quality control samples}

At the 2018 AAPS 360 Workshop on the 2018 BMV FDA guidance, it was stated that QCs expire when the material with which they were made expires. Later, this statement was clarified to say that QCs should not be prepared from expired reference material. Can it be verified that the later clarification supersedes the original statement? To avoid Form 483s, should new QCs be prepared every time the stock solution expires even if the QCs are within stability? Given the current conflict between the 2012 EMA BMV guideline [26] (frozen QCs for precision and accuracy [A\&P]) and the 2018 FDA BMV guidance [23] (fresh QCs for A\&P) what approaches are currently being used to ensure that $\mathrm{A} \& \mathrm{P}$ assessments are accepted by both regulatory agencies? Drugs typically are registered worldwide. What about for Japan MHLW, Health Canada and Brazil ANVISA? What will be the impact of the ICH M10 BMV guideline [22] if different from the 2018 FDA BMV guidance [23]? Can observations/Form 483s be reconsidered by FDA if there is discrepancy between FDA BMV and ICH M10 BMV? Please comment on the use of re-test dates versus expiration dates for materials used in bioanalytical methods. What is the appropriate application of each to meet FDA expectations?

\section{Freshly made stock solutions}

What is FDA's definition of 'fresh'? Is the GCC definition of fresh accepted by FDA? Should 'fresh' solutions be prepared for FDA studies even if the old stock solutions are within stability? What if this is in disagreement with other guidelines? To avoid Form 483s, should we prepare new stock solutions every time the reference standard material expires even if the stock solutions are within stability? What is the best approach for stability determination: (a) preparation of all stability QCs at the same time and stability determinations at selected time points (i.e., single sample preparation but multiple analyses); (b) preparation of stability QCs at selected time points and stability determinations at the same time (multiple sample preparations but single analysis)? What are the pros and cons of each approach versus the 2018 FDA BMV guidance recommendations [23]?

\section{Stability in the presence of co-medications}

Does matrix stability testing for biotherapeutics need to be performed in the presence of small organic molecules (and vice versa) as a part of the therapeutic regimen, providing the assay specificity/lack of interference is demonstrated? What are the FDA expectations for matrix stability testing for co-medications administered to the study participants in pivotal BE studies for treatment of adverse events or other health conditions, providing the assay specificity/lack of interference is demonstrated? What are the FDA expectations for stability testing in the presence of major circulating metabolites proven neither to interfere with the analyte assay nor to potentially convert to the parent 
analyte (based on the chemistry or laboratory testing)? How do you define the panel of drugs that are regularly administered for the same indication of the drug under development especially in large Phase III studies, taking patient diversity into account? What stabilities should be investigated for drugs administered as fixed combinations: just bench top and freeze-thaw as recommended by GCC or also long-term stability?

Sample management \& handling

The 2018 FDA BMV guidance [23] refers to tracking records for temperature during sample shipment. How common is the use of temperature loggers for sample shipments? How should the industry provide a record of temperature during shipments to meet FDA requirements? Where should these data be included? How has industry implemented SOPs to address requirements around sample management and handling? What is the impact on bioanalytical laboratory procedures according to industry experience?

\section{Chromatographic assays \\ Re-injection \& chromatogram reintegration}

For analytical runs requiring re-injection, are curve and QC results sufficient for the reporting requirements or do original rejected study sample results also need to be reported? For an analytical run requiring re-injection, when there are insufficient results for quantification of the original injected run, are summary sheets/results tables without curve regression or other supporting documents showing cause for re-injection acceptable to FDA for reporting requirements? For an analytical run requiring re-injection due to poor chromatography, can representative chromatograms from the original injected run showing the poor chromatography suffice for reporting requirements?

Manual integration reporting requirements are noted in 2018 FDA BMV guidance Table 1 [23]. Assuming an appropriate $\mathrm{SOP}$ is in place for manual integration procedures, under what circumstances will manual integrations be acceptable to FDA? Is this in agreement with the ICH M10 draft guideline? Should manual integrations ever be used for calibrators or QCs, or are manual integrations only acceptable for study samples with matrix interference or retention time shifts?

\section{Small-molecule endogenous analytes (or biomarkers)}

How is an appropriate and practical approach for bioanalysis of small-molecule endogenous analytes (or biomarkers) chosen? For analytes which are also endogenous, can the detailed guidelines of the ICH M10 BMV draft guideline be used [22]? Is FDA in agreement with the use of a surrogate analyte for chromatographic methods? According to the 2018 FDA BMV guidance [23], it is possible to use a fit-for-purpose (FFP) approach for biomarkers but there are no references cited. Should industry assume that FDA agrees with common FFP industry approaches to biomarkers (e.g., Lee’s paper [27] and C-Path [28])?

\section{LBA}

Anchor points

What are the best practices for demonstrating the use of anchor points? Do they meet FDA current expectations? Is this in method development, pre-validation or validation? Should anchor point evaluation be documented to avoid Form 483s, which is not the current practice? Specifically, when evaluating if they are needed, is it necessary to mathematically show various iterations with more than one anchor point? How has industry implemented SOPs to address these requirements? Have Form 483s been issued on this subject? Does the anchor point need to be above the LLOQ or below the ULOQ to be masked? Are there other objective processes for masking being utilized?

\section{Dilutional linearity replicates}

The 2018 FDA BMV guidance [23] recommends more replicates when performing dilutional linearity (five replicates in one run). The approach in the ICH M10 BMV draft guideline is significantly different [22] (one replicate in three runs). Which approach is more scientifically sound?

\section{Discussions, consensus \& conclusions}

Common topics (chromatographic assays \& LBA)

Synopsis of method development

Method development is a critical precursor to method validation and varies from extensive experimental work to develop a novel method, to simple confirmation of assay performance for a method transferred from another 
laboratory. As method validations aim to confirm all aspects of method performance, validation reports have often not provided details of method development or the origin of the method. However, the 2018 FDA BMV guidance [23] not only emphasizes the importance of method development, the Documentation and Reporting sections require a synopsis of the method development in the validation report, hence introducing a new requirement for a synopsis of method development in validation reports. Further discussions at the 2019 WRIB meeting highlighted two key points in fulfilling this requirement. Firstly, only a synopsis with limited detail is required and a formal method development report is not needed. Secondly, the primary objective is to provide key information and insights for future reviewers and users of the method. This synopsis is important because it is valuable to know the origins of the method, any special challenges that were encountered in method development, and the reasons for any unique aspects of the method. Such information can be especially helpful in troubleshooting unexpected method performance issues and understanding the reasons for revisions of a bioanalytical method across a multi-year drug development program. Examples of unique aspects that should be highlighted include specific procedures required to stabilize samples, changes in method to include or remove metabolites, or atypical approaches to analysis. References to any related validated methods should also be included (e.g., previous validation reports for the same matrix from a different species, or sponsor supplied methods). The method development synopsis serves to provide insight on possible causes when unusual or unexpected results are encountered during study sample analysis, or challenges are encountered establishing a method in a different laboratory. The exact location of the method development synopsis in the validation report is not critical; FDA only requires that it be easily located.

\section{Stability of ultra-high concentration samples}

During BMV sample stability should be demonstrated, covering the longest span of time for which a sample is stored from the time it is collected until it is analyzed. For example, a given clinical study may run for 3 years, but samples are tested in an ongoing fashion such that no study sample is stored for more than 1 year prior to analysis. In this case, demonstrated stability for 1 year would cover the longest period of time for which a sample was stored prior to analysis. Traditionally, low and high QC samples are used to perform stability evaluations. However, some recent discussions have considered the relative merits of performing ultra-high QC (UHQC) stability. It should be noted that the 2018 FDA BMV guidance does not require this evaluation.

For chromatographic assays, UHQC stability may be important in cases where low or high QC stability is marginal or fails at later time points or for some matrices where drug solubility can be an issue. In the latter case, it should be recognized that this does not indicate a stability issue but rather a technical issue with preparing the stability samples. If a calibration range is extended (higher ULOQ), the established UHQC stability could prevent the need for stability re-assessments at the new calibration range.

For LBA, dilutional linearity demonstrates that high concentration samples can be accurately diluted into assay range. Industry representatives pointed out that for large-molecules, low concentration samples are more sensitive indicators of instability, as demonstrated by the lack of examples in literature on loss of stability in high concentration samples when no instability is observed in low concentration samples. There are also concerns that aggregation or solubility issues related to the preparation of the UHQC sample may be erroneously interpreted as loss of stability. Industry representatives suggested that this evaluation does not need to be routinely performed, nor is required by the 2018 FDA BMV. Risk assessment and scientific rationale should drive the need for such evaluations.

\section{Freshly prepared calibrators \& quality control samples}

The recommendations contained within the 2018 FDA BMV guidance that indicates the use of 'fresh' calibrators and QCs in validation runs have led to much active discussion within the industry. The main issue with this recommendation is that it conflicts with the current EMA BMV guidance [26], which requires QC samples used for A\&P to be frozen. The rationale for this FDA recommendation is that stability data may not yet be available at the time $\mathrm{A} \& \mathrm{P}$ is assessed, thereby incurring risk in using frozen QCs. However, determining A\&P on multiple preparations of fresh QCs may confound control sample preparation variability with assay variability. The 2018 FDA BMV guidance indicates that fresh QCs be used for at least one A\&P assessment. Obtaining preliminary stability data during assay development may also alleviate regulatory concerns about using frozen QCs during validation.

Setting expiration dates for QC samples based on the expiration date of the reference material from which the QC samples are prepared was also discussed. The scientific rationale behind this approach was questioned, since 
the guidance document specifies that stability "is a function of the storage conditions, the physicochemical properties of the drug, the matrix and the container system. The stability of an analyte in a particular matrix and container system is relevant only to that matrix and container system and should not be extrapolated to other matrices and container systems." [23]

Discussions also clarified the differences between a re-test date and an expiration date. It is reasonable to use harmonized language in specifying that 'expired' means the product is no longer stable but a re-test date implies that stability testing is ongoing and a product could be used at the risk of the laboratory (and extended prior to completion of the study). Therefore, QC samples prepared with an expired reference standard should not be used, but QC samples can be prepared with a reference standard past its re-test date as long as stability is subsequently extended. It was also clarified that expiration or re-test dates for QCs in matrix should be based on stability data in that matrix and not based on the expiry date of the reference material. For example, if matrix QCs were prepared with reference material that will expire 6 months later, this does not limit the stability of the matrix QC to 6 months.

\section{Freshly made stock solutions}

Language in the 2018 FDA BMV guidance states that all stability determinations should use a set of samples prepared from a freshly made stock solution of the analyte. Fresh is defined as preparation on the day of the experiment [23]. This is the same language appearing in the 2001 FDA guidance [25] and the 2013 draft FDA guidance [24]. Although not a newly introduced recommendation, interpretation and implementation of this statement have varied amongst different laboratories and between LBA and chromatographic assay approaches. Some laboratories have changed procedures to ensure a fresh stock is used, while others strongly believe that using a stock solution within its stability period is scientifically sound. One concern about not using a fresh stock is that a slightly degraded stock (but acceptable - up to $10 \%$ difference is typically tolerated) could introduce a bias going into the evaluation. Those who prefer to use stable stocks believe that additional errors could be introduced in each additional stock preparation that can confound interpretation of real stability issues. Furthermore, when calibrators and QCs are made from separate stock solutions as is recommended, there is extra confidence in the reliability of the stock preparations. A consensus was reached whereby the use of both fresh stock solutions and those within the established stability period was acceptable for matrix stability evaluations.

\section{Stability in the presence of co-medications}

Discussions between regulators and industry on testing matrix stability with co-administered compounds (coformulated or specific drug regimens) started in 2010, and until 2014, no cases of stability issues were reported in the literature [29]. Until the 2018 FDA BMV guidance, regulatory expectations have been conveyed via regulatory inspections and questions arising during submission review.

Regulators' questions have focused around selectivity, potential interference with the primary analyte and a requirement for matrix stability evaluations for fixed dose combinations (FDCs). Despite data published by the GCC demonstrating that there was no effect on stability from 59 different FDCs [30], the requirement for matrix stability evaluations for FDCs and specific drug regimens remains.

A typical design used in industry involves the spiking of low and high $\mathrm{QC}$ samples with $2 \mathrm{xC}_{\max }$ levels of the co-medication.

Concomitant medication administered during a BE study

For BE studies, it has also been expected that selectivity/interference would be tested with the concomitant medications administered to study participants for treatment of adverse events and other health conditions during the conduct of the study. Regulators have stated that one of the concerns is with the ion enhancement/suppression effects. With concomitant medication the need for stability testing should be based on scientific judgment and rationale. Industry representatives proposed that it is not expected that every possible combination of drugs be tested, but that a risk assessment should be performed to determine how much testing is required based on context of use of the data. Different approaches may be justified.

\section{Sample management \& handling}

The extended requirements for sample tracking and handling in the 2018 FDA BMV guidance [23] have required the procedures in place within bioanalytical laboratories performing regulated bioanalysis to be updated accordingly. Furthermore, the new sample tracking requirements have introduced the need for improved coordination between 
the different functions of the pharmaceutical and biotechnology industries involved in managing the conduct of studies. This is particularly important for pivotal PK and BE studies which support market access, safety, efficacy and labeling endpoints. In addition, the cost implications of the new guidance should be considered when planning a new study as the new requirements around sample management will likely impact the overall cost of a study. The principal concern is the documentation of sample temperature during shipment. Some options that bioanalytical laboratories utilize include using couriers specialized in the transport of biological samples who guarantee sample integrity during transportation or employing temperature monitors that are placed in the box with the samples. Some laboratories provide QCs of proven concentration to the collection site to have shipped back with the study samples and analyzed to determine any impact of shipping conditions. In general, it is considered sufficient to use a courier that documents the refilling of dry ice and provides sufficient documentation upon receipt to prove that dry ice was still present in the box. When such a courier is not used, other means, for example, temperature monitors, may be used to support that samples were appropriately handled during transport. Moreover, for transporting of dry samples, humidity control may be just as important as temperature control. The intent of the statement in the guidance: "need to assess conditions of samples before receipt at analytical site" is that the temperature needed for sample collection and storage at the clinical site needs to be determined during method development.

\section{Chromatographic assays}

Re-injection \& chromatogram reintegration

Re-injection

Re-injection of analytical runs should be governed by suitable SOPs and scientific rationale. Equipment failure can result in the need for re-injection. When analytical runs are re-injected, a table of re-injected runs, reason for re-injection and the original and re-injected results should be reported. The proposed documentation requirements should not significantly impact the industry as most regulated bioanalytical laboratories already have appropriate applicable documentation practices in place. However, the new reporting requirements add significant work and costs to the generation and review of study reports. In addition, requirements for reporting rejected data from re-injected analytical runs could be burdensome, potentially adding hundreds of rejected sample result values to reports. Furthermore, not all rejected analytical runs can be appropriately regressed or have sample concentrations that can be properly determined. Re-injected batches should be indicated in the report whereas datasets should only be included if they are collected. Additional reported information for re-injected runs should include data used to support the rationale for re-injection as per the applicable SOP.

\section{Chromatogram reintegration}

reintegration of chromatographic data of analytical runs should be governed by suitable SOPs and scientific rationale. Despite acceptable assay performance and chromatography of a bioanalytical method during validation, subsequent studies can yield atypical analytical run performance. For instance, HPLC column to column variations can produce variable chromatographic peak shape and this can impact integrations. The 2018 FDA BMV guidance document [23] sets new requirements for documentation and reporting practices when reintegration occurs. Electronic records of original and reintegrations should be maintained, as well as the applicable audit trail. The reason for reintegration and the associated SOP for reintegration should now be included in analytical study reports (representative chromatograms in validation reports). Original and re-integrated chromatograms with results are now required in reports for BE studies. Regarding manual integrations, it has been reiterated many times that this approach is not recommended [29]. However, in cases where manual integrations are justified, the same approach should be applied consistently across the study. All data, prior to and after reintegration, should be included in study reports.

\section{Small-molecule endogenous analytes (or biomarkers)}

Biomarkers are endogenous molecules in biological matrices. The 2018 FDA BMV guidance [23] stresses that "it is critical to ensure the integrity of the data generated by assays used to measure them. Biomarkers can be used for a wide variety of purposes during drug development; therefore, a fit-for-purpose (FFP) approach should be used when determining the appropriate extent of method validation." The guidance elaborates on the FFP concept, stating that the level of validation should be appropriate for the intended purpose of the study. Although the guidance indicates that similar BMV philosophies should be used as a starting point for BAV, particularities of assaying biomarkers may mean that some requirements may not be appropriate or some should be added based on the context-of-use [28] of a specific biomarker. The specific expectations of the FDA regarding BAV are that the biomarker section of the 
guidance was intentionally brief and any appropriate approach can be used as long as it is properly justified and demonstrates good science.

\section{LBA}

Anchor points

Anchor points are useful in calibration curves of many ligand-binding assays in order to better define the non-linear curve fit. Sensitivity can be increased by including anchor points at the lower end of the calibration curve. Since anchor points should not be used in the assessment of the standard curve acceptance criteria, masking/exclusion criteria for anchor points are generally not addressed in guidance documents [31]. Having detailed SOPs to ensure an unbiased approach for masking of calibrators and anchor points is helpful to avoid observations and potential issuance of health agency findings. There was consensus that if an assay demonstrates problems with anchor points, this might be indicative of an issue with the assay itself or that the anchor point is not necessary. It was clarified during discussions that the intent of the statement in Table 1 of the guidance, which states "anchor points should not be included in the curve fit" is that "anchor points should be used to fit the curve but should not be used to count the minimum number of acceptable standard levels."

\section{Dilutional linearity replicates}

Despite significant advances in LBA technologies, the quantitative range for LBA assays remains limited, generally one to three orders of magnitude. Analyzing samples of high concentration using LBAs often requires significant sample dilution. Therefore, demonstrating dilutional linearity is critical for reliable measurement of study samples. Industry best practices recommend that high concentration sample(s) be prepared in matrix at concentrations above the assay range, including at the anticipated $\mathrm{C}_{\max }$ or the highest possible concentration that can be achieved with the available reference standard while maintaining $95 \%$ matrix $[9,12,15,18,21,62]$. These samples are serially diluted to cover the entire quantitation range. Typically, at least one sample above ULOQ (to evaluate hook effect), three to five samples spread across the quantitation range, and one sample below LLOQ are included for each dilution series and run in the assay. Three independently prepared dilution series are run in the assay with the expectation that at least two out of three replicates must pass assay acceptance criteria (accuracy $\pm 20 \%$ of nominal, precision $<20 \%$ CV). In addition, cumulative precision of $<20 \%$ is commonly applied. Cumulative precision refers to the precision of dilution-adjusted concentrations that are within the quantitation range. In contrast, the 2018 FDA BMV guidance requires five replicates for each dilution factor and states $\pm 20 \%$ criteria for accuracy and $<20 \%$ precision. The guidance does not state the need for placing dilutional linearity samples across the quantitation range of the assay. The acceptance criteria do not include the number of acceptable replicates nor cumulative precision.

This topic generated much discussion since performing the evaluation for every dilution factor used (or anticipated) in sample analysis is burdensome versus successfully testing the highest dilution factor to demonstrate that the range of dilution factors used in study sample analysis has been covered. However, it was explained that the rationale for the recommendation stemmed from regulatory inspections that found method and analyst technical issues that cast doubt on the reliability of the data. Industry representatives noted, however, that issues related to analysts' technical ability to dilute accurately should be addressed via improved training practices rather than be incorporated into specific method validations. Regulators recommended that five replicates per dilution factor should be tested. The dilution factors used during sample analysis shall be within the dilution factors tested in method validation. The number of replicates per factor was selected to mimic the A\&P evaluation.

\section{Recommendations}

Below is a summary of the discussions on the 2018 FDA BMV made during the $13^{\text {th }}$ WRIB expert panel.

\section{Common topics (chromatographic assays \& LBA)}

1. A formal method development report is not required, a discussion in the validation report outlining the life cycle of the method is sufficient. Unique aspects of method development and reference to related validated methods should also be included;

2. Ultra-high concentration stability (note this is not an FDA BMV requirement);

- For LBA, the ultra-high concentration stability evaluation does not need to be routinely performed. Risk assessment and scientific rationale should drive the need for such evaluations; 
- For chromatographic assays, UHQC stability may be important in cases where low or high QC stability is marginal or fails at later time points or for some matrices where drug solubility can be an issue. For some studies, stability assessments using UHQCs should obviate the need for re-validation of matrix-based stability at multiple calibration curve ranges;

3. Fresh QCs should be used for at least one A\&P assessment;

4. 'Expired' means the product is no longer stable. A re-test date indicates that stability testing is ongoing, and a product could be used at the risk of the laboratory;

5. QC samples prepared with an expired reference standard should not be used, but QC samples can be prepared with a reference standard past its re-test date as long as stability is subsequently extended;

6. Both fresh stock solutions and those within the established stability period can be used for matrix stability evaluations;

7. A risk assessment should be performed to determine what stability testing in the presence of co-administered compounds is required for FDCs based on context of use of the data. The minimum recommended testing is provided in the guidance, although different approaches may be justified. The recommended experimental design in industry is spiking of low and high $\mathrm{QC}$ samples with $2 \mathrm{xC}_{\max }$ levels of the co-medication;

8. Use of a courier that documents the refilling of dry ice and provides sufficient documentation at reception to prove that dry ice was still present in the box is considered adequate documentation. Temperature monitors are appropriately used during sample shipping when such a specialized courier is not employed;

9. Sample collection procedure and storage conditions (e.g., temperature and protection from light) at the clinical site needs to be determined during method development or validation.

\section{Chromatographic assays}

1. Re-injected batches should be indicated in the report. Datasets should only be included if they are collected. Additional reported information for re-injected runs should include examples of data used to support the rationale for re-injection;

2. Manual integrations are not encouraged. If used, they must be justified and applied consistently across the study. All data, prior to and after reintegration, should be included in study reports;

3. An FFP approach to BAV is recommended with proper scientific justification.

LBA

1. A detailed SOP to ensure an unbiased approach for masking of calibrators and anchor points is recommended;

- Anchor points should be included when needed to facilitate curve fit but are not subject to acceptance criteria. Use of anchor points should be limited to one near the LLOQ and one near the ULOQ;

2. The FDA recommends that five replicates per dilution factor should be tested for dilutional linearity. Factors should mimic those used in sample analysis. The number of replicates per factor was selected to mimic the A\&P evaluation.

\section{Section 2 - Feedback on ICH M10 BMV draft guideline}

Lauren Stevenson ${ }^{13}$, Olivier Le Blaye ${ }^{22}$, Michael Buonarati ${ }^{3}$, Matthew Andisik ${ }^{15}$, Chad Briscoe ${ }^{16}$, Stephanie Cape $^{17}$, Arindam Dasgupta ${ }^{1}$, Sally Fischer ${ }^{18}$, Fabio Garofolo ${ }^{5}$, Sam Haidar ${ }^{1}$, Roger Hayes ${ }^{19}$, Rafiq Islam ${ }^{6}$, John Kamerud ${ }^{21}$, Gustavo Mendes Lima Santos ${ }^{23}$, Corey Nehls ${ }^{24}$, Christina Satterwhite ${ }^{11}$, Natasha Savoie ${ }^{12}$, Catherine Soo ${ }^{25}$, Sriram Subramaniam ${ }^{1}$, Nilufer Tampal ${ }^{1}$, Theingi Thway ${ }^{1}$, Stephen Vinter ${ }^{26}$, Emma Whale ${ }^{26}$, Keyang $\mathrm{Xu}^{18}$

Authors are presented in alphabetical order of their last name, with the exception of the first three who were major contributors and/or notetakers. Author affiliations can be found at the beginning of the article.

Note: Brian Booth (FDA), Jan Welink (EMA), Anna Edmison (Health Canada), Akiko Ishii-Watabe (MHLW), Yoshiro Saito (MHLW) and Thais Correa Rocha (ANVISA) from the ICH M10 EWG helped, organized, coordinated and participated in the ICH M10 discussions and activities throughout WRIB. Their purpose was to attend WRIB, provide clarity on ICH M10 and seek public input on the draft document. The ICH M10 EWG neither endorses nor refutes any of the below recommendations, nor are they authors of these remarks due to conflict of interest.

An excerpt from the Decennial Index [29] suitably highlights the efforts for a single harmonized BMV guideline:

"One of the major impacts WRIB has had in the field of bioanalysis has been the effort to globally harmonize bioanalytical method validation and sample analysis. In 2010, the consensus was reached that global harmonization of bioanalytical guidance is an important and necessary step in facilitating global submissions of studies supporting the release of new drugs. There was a call supported by US FDA at the 2010 WRIB to create an all-inclusive Global Bioanalytical Consortium with representation from scientific associations worldwide to discuss harmonization of bioanalytical regulatory 
guidance and ideally propose unified guidance language that can be presented to regional agencies [3]. This group was created and a consensus was attained at the 2011 WRIB and resultant White Paper: the pursuit of consistency among regulatory inspectors can be facilitated via appropriate training, meetings and constructive exchanges among countries [4]. The discussion on global harmonization of BMV guidance/guidelines continued in 2012 when the regulatory agencies' effort toward bioanalytical global harmonization was recognized by having multiple, but harmonized, bioanalytical guidance documents/guidelines" [5].

Indeed, the ICH launched the creation of a harmonized BMV guideline (M10) with an expert working group in 2016 [32]. The first draft of the M10 BMV guideline was published for public comment in February 2019 [22].

Discussion topics \& consolidated questions collected from the global bioanalytical community Overall, the ICH M10 BMV draft guideline [22] has been positively received by the global bioanalytical community attending the $13^{\text {th }}$ WRIB, and only a few major topics were identified which required further discussion. The following paragraphs report the consolidated questions collected from the global bioanalytical community. Hence, the discussion topics were extracted from these questions and considered the most relevant 'hot topics' for the ICH M10 guideline discussions. They were reviewed and consolidated by internationally recognized opinion leaders before being submitted for discussion during the $13^{\text {th }}$ WRIB. Background information on the issues, discussion summaries, consensus and conclusions are in the following section and a summary of the key recommendations is provided in the final section of this manuscript.

Corrections such as clarifications, redundancies, discrepancies and typos were considered minor issues. They were brought up during the expert panel working dinners and/or during the three open forums and effectively discussed since they can be easily addressed by the EWG during the final revision of the document. The most interesting and thought provoking of these issues are listed after the main recommendation in the section entitled 'Minor Issues \& Suggestions'.

\section{ICH M10 BMV draft guideline - chromatographic assays Hybrid LBA/LCMS}

Lack of regulatory guidelines is currently producing confusion when hybrid LBA/LCMS methods are used in regulated bioanalysis for PK assays in support of clinical trials. What are the regulatory expectations for hybrid assay BMV? Real world clinical PK data confirm the recommendations of the 2015-2018 White Papers in Bioanalysis $[10,13,16,19]$. How are these recommendations translated in the ICH M10 draft guideline? Do you agree that the ICH M10 guideline should at least ask that the following be included in the BMV if hybrid LBA/LCMS assays are used?

- Justification for using hybrid LBA/LCMS rather than LBA;

- Risk of data discrepancies with prior method;

- Demonstration of the selectivity and exclusivity of the signature peptide;

- Demonstration of the impact of completion of enzymatic digestion vs recovery;

- Demonstration of internal standard (IS) tracking for hybrid LBA/LCMS methods.

\section{Medium QC placement}

The ICH M10 draft guideline [22] has no indication that 30-50\% should be interpreted on a geometric scale rather than arithmetic. Is current ICH M10 draft guideline wording clear enough for arithmetic medium QC placement or does it need further clarification? Does the ICH M10 draft guideline need further clarification on the routine strategies used by industry in placing additional QC levels to ensure that two QC levels fall within the range of study sample concentrations?

\section{IS variability}

Internal standard (IS) variability has been a very hot topic in recent industry/regulator discussions as an important part of BMV and sample analysis monitoring. Does the ICH M10 draft guideline need further clarification on regulatory requirements for IS variability? Are recent regulatory concerns on the extent of IS investigations/CAPA performed by the industry clearly addressed in the ICH M10 draft guideline? What are the regulatory expectations for underlying causes for IS variation and its impact on quantitation? Can harmonization be achieved regarding criteria for IS variation (e.g., is $80-120 \%$ acceptable?), performing adequate monitoring of IS across 
runs/batches/studies, data interpretation, identification of anomalies and troubleshooting? Should the ICH M10 guideline consider previous White Paper recommendations on this topic $[10,16]$ ?

\section{Matrix factor}

The ICH M10 guideline [22] has no reference to performing matrix factor. Is there value in this evaluation? Should it be added?

\section{ICH M10 BMV draft guideline - LBA \\ Parallelism}

Parallelism for LBA PK assays as part of BMV has always been a controversial topic. It became even more controversial since the 2012 EU EMA BMV guideline [26] was issued asking to routinely perform parallelism. Is there agreement that parallelism for PK assays is best implemented to investigate anomalous study data and should not be a routine validation assessment? If yes, do you agree that a clear and harmonized ICH M10 guideline recommendation on parallelism should include:

1) A statement to clarify that parallelism in BMV should be performed only when needed;

2) A justification about why it is not performed so that the regulators are well informed on why it was not performed;

3) A harmonized justification template when parallelism is deemed unnecessary in validation;

4) When parallelism is needed (in some cases, parallelism may be needed for each study), the $30 \%$ criterion may be used but with a justification to ensure that there are no trends of non-parallelism;

5) A statement mentioning that the parallelism evaluation, when needed, should be included with sample analysis rather than with method validation?

\section{Single-well analysis}

Are there any remaining regulatory concerns about moving forward with a single-well format? How can the ICH M10 guideline provide flexibility and/or clearer guidance on this topic? What acceptance criteria in the ICH M10 guideline would be most appropriate for single-well analysis? Although samples are run in single well, should standards and QCs continue to be run in duplicate? If standards and/or QCs are run in a single well, can masking of outliers still be performed and what are the rules around such masking? Is continuing to run the standards/QCs or just QCs (i.e., two independently prepared single-well sets) in duplicate justified, or could this bring potential concerns for regulators that samples are treated differently than controls? Can the ICH M10 guideline provide guidance on plate layout for single-well analysis to meet regulatory expectations? What is the potential for increased plate failure if we were to run the standards and QCs in single well? Is this concern outweighed by the potential to increase plate output by more than twofold? How do we strike a balance between using the QCs to mimic the samples versus having a specific number of QCs on each plate that is representative of the sample count? Does plate location of the QCs matter?

\section{Replicate analysis}

Common practice in LBA is to have calibrators, QC and study samples in duplicate (or even triplicate) to minimize LBA variability by plating the same sample into two adjacent wells. If duplicate analysis is used, QC and study sample results are reported as a mean of two values. Is the duplicate/replicate wells approach a way to minimize variability close to LLOQ, to identify random errors or both? Can the ICH M10 guideline provide harmonized criteria and guidance on how to handle duplicate/replicate wells? What are the acceptance criteria for study samples analyzed in duplicate wells? Should there be precision criteria $(\% \mathrm{CV})$ for intra-replicate values (i.e., between wells)? Does the \%CV of two points make statistical sense? Is it better to use triplicate wells to identify outliers? Should this \%CV be calculated using response or concentration, or both? What is the impact of calibration curve shape on the method used (response or concentration)? What if the precision using back-calculated concentrations passes the $20 \%$ criteria but it does not pass if raw signal is used or vice versa? Is it acceptable to deactivate a single replicate when running in duplicate? Should we mask both duplicate wells to avoid bias? Can the ICH M10 guideline provide well-defined acceptance criteria to eliminate replicate wells when there are more than three? When should study samples be repeated due to major well-to-well difference in responses? 


\section{Interference impact}

Is the ICH M10 draft guideline [22] language enough to clarify regulatory expectations on the interpretation of factors that impact LBA PK assays for measurements of biotherapeutics? How do the presence of high concentrations of circulating target, assay reagents, format and conditions significantly affect LBA PK assay performance and results for PK parameters/profiles? Is the ICH M10 guideline language sufficient for clarifying regulatory expectations on estimation of interference impact due to soluble versus receptor targets or total versus free assays? How should ADA interference in LBA PK assays be analyzed and reported? What is the recommendation of the ICH M10 guideline on this type of outcome and expectations (e.g., general analysis description, findings and interpretation, etc.)?

\section{Stability of ultra-high concentration samples}

(Excerpt from Section 1 - this topic was discussed for both 2018 FDA guidance and 2019 ICH M10 BMV draft guideline):

"Must $>U L O Q$ QCs used for stability evaluations bracket study sample concentrations or can they reside near to where the majority of $>U L O Q$ study sample concentrations are observed? If a stability interval passes for low and high QCs, but fails at the $>U L O Q Q C$, can a single re-analysis stability assessment be used to establish stability if acceptance criteria are subsequently met for the $>$ ULOQ QC stability samples (e.g., previous failure likely due to dilution processing error)? Is there alignment that low concentration samples are most sensitive indicators of stability for most large-molecule and nucleic acid therapeutics? What are specific special cases where evaluation of high concentration samples is supported by scientific rationale?"

\section{Stability of biotherapeutics at both $-20^{\circ} \mathrm{C} \&-70^{\circ} \mathrm{C}$}

Why is it necessary to perform additional long-term stability evaluations of biotherapeutics at $-70^{\circ} \mathrm{C}$ once stability at $-20^{\circ} \mathrm{C}$ is established when there is no scientific data indicating an issue?

\section{ICH M10 BMV draft guideline - common topics \\ Whole blood stability}

Why is it not practical and scientifically meaningful to develop an exploratory method in blood if your official bioanalytical method is in plasma or serum? What are the practical issues to developing an exploratory method in whole blood versus an already validated method in plasma or serum (e.g., ion suppression, reduced sensitivity and recovery, more complex extraction procedure, chromatographic conditions, red blood cell (RBCs) lysis, etc.)? What does exploratory method mean in regulated bioanalysis? What is the reliability of an exploratory method for a validation test? Are these exploratory results reliable and accepted by regulators for supporting whole blood stability in clinical studies? Whole blood stability testing has already been globally harmonized among CROs [33]. Can this approach be specified in the ICH M10 guideline for clarity?

\section{Lipemic matrix testing}

Why is natural plasma with a triglyceride concentration of $300 \mathrm{mg} / \mathrm{dl}(3.40 \mathrm{mM})$ or greater the most appropriate hyperlipidemic matrix to use in BMV [61]? Why should the potential use of artificial lipemic matrix (i.e., matrix mixed with fat emulsion) be removed from the ICH M10 guideline? Lipemic matrix testing has already been globally harmonized among CROs [34]. Can this approach be specified in the ICH M10 guideline for clarity? Can the ICH M10 guideline standardize the information requested of vendors of lipemic matrix by requesting a lipemia index as a requirement of the plasma certificate and the minimum requirements for the lipemia index?

\section{Robustness}

'Validation of a robust/rugged bioanalytical method' is very often claimed in bioanalytical publications and reports. Is this just a casual statement or are specific experiments performed to support this claim? Should the claimed robustness of bioanalytical methods be demonstrated formally, in addition to the tests already listed in the ICH M10 draft guideline [22]? If yes, can a list of tests be defined and agreed upon, or should this be a case-by-case determination, based on the method characteristics and intended use? If a list of predetermined tests needs to be defined, what are these tests in BMV to ensure method robustness based on precision and accuracy: multiple instruments, multiple columns lots, multiple analysts, representative batch size? Robustness has already been harmonized among CROs $[33,35]$. Can this approach be adopted in the ICH M10 guideline? 


\section{Certificates of analysis}

Quality of the reference material directly affects the reliability of the bioanalytical method. Should the ICH M10 guideline have more details specifically on certificate of analysis (CoA) requirements? Is current ICH M10 draft guideline [22] wording adequate for CoA harmonization? Do you agree that clear and harmonized ICH M10 guidelines on reference standards should include comprehensive stability data, clear and detailed shipping conditions, harmonized information and CoA templates from different suppliers, stability data for metabolite reference materials, harmonization on minimum requirement for reference standard characterization? Are the ICH M10 guideline recommendations on life cycle of reference material adequate; that is, expiration date, re-testing and re-characterization, standardization of correction factors by including the correction factor and the calculation used to arrive at the correction factor in the CoA? Stock comparison has already been harmonized among CROs [36]. Can this approach be adopted in the ICH M10 guideline?

\section{Discussions, consensus \& conclusions}

$\mathrm{ICH}$ M10 BMV draft guideline - chromatographic assays Hybrid LBA/LCMS

Over the years hybrid LBA/LCMS has gained increasing acceptance in the drug development industry as a complementary, alternative, or even a preferred, bioanalytical approach to conventional LBA for quantification of protein therapeutics and protein biomarkers $[10,13,16,19]$. With the hybrid LBA/LCMS data reported so far positively demonstrating the robustness of assay performance, bioanalytical scientists are motivated to expand its application in the regulated environment. Although guiding principles for validation of hybrid LBA/LCMS assays have been actively discussed and proposed among the experts in the field, the current issued guidance documents from US FDA [23] or EMA [26] have not addressed key specifications/requirements for this category of assays. To better enable the support of clinical trials, emphasis has been placed on how to establish appropriate acceptance criteria around precision and accuracy for hybrid LBA/LCMS assays. Due to the greater complexity involved with hybrid LBA/LCMS assays, the LBA acceptance criteria of 20/25 were initially adopted and used in most situations of protein quantification instead of the 15/20 rules defined for typical chromatographic methods of small molecules. Industry representatives proposed adding the minimum requirements for hybrid LBA/LCMS assays to the ICH M10 guideline as a starting point to help the industry move forward in the implementation of this bioanalytical platform in regulated bioanalysis when deemed complementary and/or alternative to traditional LBA.

Experts in hybrid LBA/LCMS assays identified the following fundamentals that should be addressed in the guidance:

- Specificity of the surrogate peptide(s);

- Digestion efficiency;

- LBA (20\%/25\%) accuracy, precision and 30\% ISR criteria should be targeted until more experience with hybrid assays has accrued;

- Dilutional linearity;

- Capture reagent lot-to-lot variation.

\section{Medium QC placement}

The ICH M10 BMV draft guideline [22] indicates a minimum of four QC concentration levels covering the calibration curve range should be included during method validation, that is, at the LLOQ, within three times the LLOQ (low QC), around 30-50\% of the calibration curve range (medium QC), and at least at $75 \%$ of the ULQ (high QC). Similar language was used in the EMA BMV guideline [26]. Although in the EMA's Overview of Comments document [37] it is stated that the arithmetic value should be used and that an applicant is free to add additional QC concentration levels, in some recent submissions it has been observed that some analytical laboratories apply a different approach when selecting the concentration level of the medium QC. Instead of using the arithmetic value, the geometric mean is used for the medium QC concentration level. This often results in the medium QC level being too low in the calibration curve range. This issue is directly related to using an adequate calibration curve range for study samples. If sample concentrations are clustered, then the range may not be appropriate. Use of a geometric mean for the medium QC can sometimes result in too large a span between the mid and high QCs and is thus considered of concern, especially in the case of $\mathrm{C}_{\max }$ values falling around $50 \%$ of the calibration curve range. It was concluded that the placement of the mid QC between 30 and 50\% of the 
ULOQ concentration as per the current guideline should be the standard, especially for chromatographic assays in which the calibration curve range is wide. An adequate number of QC levels should cover the full range of study samples, not just be placed anywhere within the profiles.

\section{IS variability}

Internal standards are used in LCMS methods to normalize variability caused by sample processing, matrix and instrumentation. In theory, a constant IS response is indicative of consistent sample preparation and LC-MS/MS method robustness. Some differences in the way IS response variations are monitored and addressed are seen across analytical laboratories, making this a relevant topic for chromatographic assays. White Papers consistently recommend that SOPs should be in place to address internal standard variability, and that trends should be investigated [10,16]. The ICH M10 BMV draft guideline [22] contains language considered too general/simplified regarding IS variability, without providing specific guidance on when a formal investigation should be initiated. It was concluded that ICH M10 wording should be improved to better address that a trend in IS variability should be investigated. Sample internal standard response should not be significantly different from the response for the calibration standards and QC samples. If significant differences are observed this should be investigated to determine if there is an impact on sample concentrations.

\section{Matrix factor}

The ICH M10 BMV draft guideline [22] provides recommendations on performing the matrix effect evaluation by spiking low and high QC samples in six different lots of matrix. The guideline presents criteria for accuracy and precision for the matrix effect samples that must be met in all individual lots. However, the guideline appears to be open to different interpretations and it is not clear whether the accuracy and precision criteria are for individual lots, or for the overall mean of the lots. It is recommended that it be clarified that the accuracy and precision criteria be applied to each of the individual lots. Evaluating the matrix factor was introduced in the EMA BMV guideline [26]. The order of magnitude of absolute matrix factor can be considered common practice as part of method development. However, matrix factor also provides important information on relative matrix effects, which could affect the accuracy of the LLOQ. Hence, it is recommended that peak areas of spiked QCs should also be reported to show lot-to-lot variations on analyte responses.

\section{ICH M10 BMV draft guideline - LBA Parallelism}

Over the last several years, routine performance of parallelism for LBA PK assays has been a topic that has generated diverse opinions that have fueled robust scientific debate $[5,6,9,12]$. There is lack of alignment on if, and when, this assessment should be performed (despite its inclusion in some regulatory guidance [26]), and the $30 \% \mathrm{CV}$ criterion often applied and recommended by the ICH M10 BMV draft guideline [22], may not sufficiently demonstrate lack of parallelism. In order to determine the value of assessing parallelism, it is necessary to understand the information the results provide. For biomarker methods (that are outside of the scope of M10), parallelism is conducted to understand characteristics of analytes from different sources, set the MRD, set the LLOQ for endogenous analytes and assesses assay selectivity $[27,28,63]$. Therefore, it is more appropriately executed during method development. For PK methods, parallelism can aid in data interpretation when confounding interferences are present in vivo [38].

In Section 7.2 of the ICH M10 draft guideline, Parallelism, it is already stated that "...parallelism of LBA should be evaluated on a case-by-case basis, e.g., where interference caused by a matrix component (e.g., presence of endogenous binding protein) is suspected during study sample analysis. Parallelism investigation or the justification for its absence should be included in the bioanalytical report." During the discussions, industry representatives confirmed that for PK methods, parallelism should be implemented to investigate anomalous study data and should not be a routine validation assessment. The ICH M10 draft guideline states that the justification for the absence of a parallelism evaluation should be included in the bioanalytical report [22]. It is recommended that the language be changed to require a justification of its applicability and an explanation of the results presented. Regarding the acceptance criteria, industry representatives questioned whether the criteria were an appropriate indication for detecting trends in parallelism. Since it is an investigative tool for PK assays, it was suggested that no a priori criteria are required. 


\section{Single-well analysis}

While single-well analysis has been widely established as the standard in clinical diagnostic and LCMS laboratories, this practice has not been routinely adopted by the regulated bioanalytical community for LBAs. Over the years, regulators have been willing to consider the application of a single-well analysis approach in LBA method validation and sample analysis $[5,9,15,29]$, but the burden of proof lies with pharmaceutical companies and CROs to demonstrate suitability. Current industry practice is to utilize 'conventional duplicate analysis', where the same sample is pipetted into two adjacent microplate wells. With improved pipette precision and the integration of automated systems, the reproducibility between wells has been less of a concern. It has been demonstrated that the intra-assay variability within a single plate is less significant than the inherent inter-assay variability (e.g., day-to-day, analyst-to-analyst or batch-to-batch) present in traditional LBAs. In addition, there are other widely adopted processes in place such as outlier identification/repeat analysis and incurred sample reanalysis (ISR) to mitigate any perceived risks in conducting single-well analysis.

The ICH M10 BMV draft guideline allows for the use of one (or more) wells per sample. However, the guideline does not clarify if the acceptance criteria for LBAs are different if single-well analysis is used. It is recommended that the criteria should be the same irrespective of the number of wells used per sample. As long as the method is validated using single-well analysis, then it is acceptable for use. In addition, if single wells are used for the analysis of samples, single wells should also be used for the analysis of calibration standards and QCs. It is further recommended that duplicate QCs per level be used for analysis and the industry practice is to place one of each at the front and back of each plate.

\section{Replicate analysis}

For LBA assays using replicate analysis (typically in duplicate), each sample is pipetted into two wells and the reported result is the mean of the two wells. Although generally accepted as artificial as \%CV cannot be meaningfully determined with only two values; the use of two values to calculate $\% \mathrm{CV}$ between wells is the industry-accepted procedure when using replicate analysis. Most laboratories establish acceptance criteria for precision of these replicates and reject a result if the \%CV exceeds some predetermined limit. For this purpose, some laboratories calculate $\% \mathrm{CV}$ of the response values between the two wells, while other laboratories use the interpolated concentration values to calculate precision. The inter-well acceptance criteria and the method of calculating them are not described in current FDA BMV guidance [23] or EMA BMV guideline [26].

Under Section 4.2 of the ICH M10 draft guideline, Validation, it is indicated that if multiple wells per sample are used, the reportable sample concentration value should be determined either by calculating the mean of the responses from the replicate wells or by averaging the concentrations calculated from each response. Data evaluation should be performed on reportable concentration values [22]. It is recommended to specify in the ICH M10 that a priori criteria should be defined in SOPs and validated. One well should not be deactivated in order to accept a calibrant level, QC or the sample. If the precision between wells does not meet the predefined precision criteria, the sample should be repeated.

\section{Interference impact}

PK assessments are critical in all phases of therapeutic biologic development. Since antibody therapeutics are bivalent, when they engage with targets they can exist in different forms including fully bound, partially bound, or unbound forms. In the majority of cases where the circulating target levels are low, or when antibody therapeutic concentrations are maintained at higher levels than their therapeutic target, the 'total' and 'free' drug levels are practically equivalent. However, in the presence of high levels of circulating target, different complexes can coexist and 'total' and 'free' drug concentrations may differ significantly. In addition to circulating targets, anti-drug antibodies can bind to the drug and impact the accurate measurement of therapeutic biologics. Depending on the choice of assay format and conditions, the PK parameters/profiles for an antibody therapeutic can be significantly different in the presence of high levels of circulating target or ADA. Therefore, understanding the assay as well as the strengths and limitations of the resulting data are crucial for data interpretation and for drawing accurate conclusions.

The discussion of this topic revolved around the language used in the ICH M10 BMV draft guideline. The LBA PK assay strategy for measurements of biotherapeutics is critical especially in the presence of a large concentration of circulating target. In these instances, the strategy should be based on biology and the rationale for what drug 
species to measure, the anticipated target concentrations and technical challenges and practical limitations of the assays and reagent availability. The estimation of the impact of interference due to circulating targets and total vs free assays should be assessed using the molar ratio of any potential interfering molecule to drug based on what is expected during validation. Note that post-dose target levels are usually much higher than baseline levels; therefore, the post-dose levels should be used to calculate target to drug molar ratios during validation. When analyzing and reporting on ADA interference in LBA PK assays, PD biomarkers can be a useful tool to assess impact of ADAs on target engagement when available.

\section{Stability of ultra-high concentration samples}

This topic was extensively discussed in the prior section on the implementation of the 2018 BMV guideline (see Section 1) and was reiterated during the open forums and expert panel working dinner for the ICH M10 BMV draft guideline. The industry representatives proposed the following for both 2018 FDA guidance and 2019 ICH M10 BMV draft guideline (excerpt from Section 1):

- "For LBA, the ultra-high concentration stability evaluation does not need to be routinely performed. Risk assessment and scientific rationale should drive the need for such evaluations;

- For chromatographic assays, ultra-high QC stability may be important in cases where low or high QC stability is marginal or fails at later time points or for some matrices where drug solubility can be an issue. For some studies, stability assessments using ultra-high QCS should obviate the need to re-validate matrix-based stability at multiple calibration curve ranges."

Moreover, since no LBA data have been published to indicate that there is instability at ultra-high concentrations that would not be seen at the low QC level, industry representatives suggested removing the sentence "the concentration of the stability QCS should be adjusted, considering the applied sample dilution, to represent the actual sample concentration range." It was confirmed in Section 1 that for LBA methods, the evaluation of high concentration QC stability to account for the study sample concentration range does not need to be performed routinely. Such an assessment should be performed only as needed based on scientific rationale.

\section{Stability of biotherapeutics at both $-20^{\circ} \mathrm{C} \&-70^{\circ} \mathrm{C}$}

Industry representatives confirmed assessing stability at two different temperatures is not necessary. Since no LBA data have been published that shows a protein is less stable at $-70^{\circ} \mathrm{C}$ than at $-20^{\circ} \mathrm{C}$, the requirement to perform stability evaluations at both temperatures should be removed.

\section{ICH M10 BMV draft guideline - common topics}

Whole blood stability \& lipemic matrix testing

The benefits of plasma and serum classify them as the preferred matrices for bioanalysis. Because bioanalytical method validation should demonstrate accuracy of concentration for incurred study samples, whole blood stability and lipemic matrix evaluations have been accepted as important experiments in proving the validation of a bioanalytical method.

Whole blood stability mimics the conditions in which plasma samples are processed in the clinic, while the lipemic evaluation ensures the robustness of the method for subjects with hyperlipidemia, high triglyceride levels or following a high-fat meal. Harmonization around the experimental designs and criteria of both tests is paramount in assuring consistent whole blood stability and lipemic evaluations across bioanalytical laboratories.

\section{Whole blood stability}

There are some challenges when testing whole blood stability. For example, the developed method has been optimized for plasma or serum and is likely not optimal for the analysis of whole blood. Moreover, purchased whole blood is not as fresh as incurred samples, and artificially incubating to encourage partitioning and binding may not be representative of what is happening in the body. Add the fact that whole blood used for this evaluation must contain anticoagulant, and the evaluation for a serum-based assay becomes impossible to perform.

Therefore, it is recommended for plasma assays to change the ICH M10 wording to clarify that whole blood stability should be initially performed in method development [2] to assess whether nonspecific binding is present 
versus an actual stability issue and, if no issues are found, then to confirm the test in validation. The whole blood stability should be performed using the plasma assay for the experiment as described in detail by GCC [33]:

“... sufficient aliquots of whole blood are fortified and equilibrated. Time zero (to) samples are drawn and are then immediately centrifuged to harvest plasma. The remaining blood samples are maintained at the desired test condition(s), and then aliquots are drawn and centrifuged to harvest plasma. The plasma aliquots are analyzed simultaneously and compared to assess stability for the desired time period..."

A method in whole blood should only be used during investigations if the plasma assay fails in order to understand if there is an actual stability issue, for example, to differentiate from a slow partitioning between plasma and red blood cells. The term "exploratory whole blood method" in the ICH M10 is misleading and should be removed or replaced with 'reliable whole blood method' to ensure that the whole blood method used for the investigation is precise and accurate and key method development parameters/experiments are tested/performed (for instance, for chromatographic assays: ion suppression, sensitivity and recovery, extraction, chromatographic conditions or impact of red blood cell [RBCs] lysis). Furthermore, it was recommended that the ICH M10 guideline should be adjusted so that the evaluation for serum is not required.

\section{Lipemic matrix testing}

The ICH M10 draft guideline discusses the need to perform selectivity in lipemic samples [22]. Natural matrix is recommended in agreement with industry standards [39,61]. However, it also allows bioanalysts to prepare hyperlipemic matrix by spiking as necessary even if this approach is considered not representative of incurred samples.

It is recommended not to use artificial lipemic matrix under any circumstances and to remove the sentence: “. . it is acceptable to spike matrix with triglycerides even though it may not be representative of study samples. "Natural lipemic matrix from donors with a good CoA is readily available. Furthermore, it is recommended to add to the guideline the use of certified hyperlipemic matrix with a CoA indicating at least $300 \mathrm{mg} / \mathrm{dl}$ triglyceride levels (lipemic index) or even higher if the study is in a special population with such high levels. Similar recommendations are already used in industry [39,61].

\section{Robustness}

Robustness is defined in ICH Q2 [40], but not in the EMA, FDA or ICH M10 BMV guidance documents [22,23,26]. The guidance lists some tests to be performed which could be considered to be part of the demonstration of the robustness of a method but may not be sufficient depending on the circumstances. The robustness of an analytical method is commonly defined as an assay's ability to withstand common daily variations in standard laboratory conditions including minor changes in mobile phase, analysts, incubation time, temperature and reagents; both critical and routine.

For chromatographic assays, it was concluded that robustness is an operational issue already demonstrated by between-run A\&P and ISR and so no additional guidance is needed.

For LBA, it was noted that robustness evaluations are often included in method development. Inclusion of formal robustness testing in method validation should be based on a risk analysis of the method taking into consideration the critical steps of the assay, the size and the length of the study and what had already been demonstrated during method development. If performed, a balanced design for robustness, testing of all the extremes together, is recommended. If both extremes (e.g. longest vs shortest incubation times) meet criteria, then robustness is considered to have been demonstrated for all intermediate conditions.

\section{Certificates of analysis}

The quality of reference materials directly affects the reliability of the bioanalytical method. Therefore, harmonization of certificates of analysis is an important step in moving forward with global harmonization of bioanalytical assays. There are still many challenges with obtaining adequate and consistent information on CoAs that can be addressed by the bioanalytical industry. These include purity and stability, re-testing versus expiry dates and correction factors. There are several ways that CoAs for reference standards are found to be inadequate; viz, lack of stability data or information on shipping conditions, differences in templates from different suppliers that can lead to interpretation challenges, missing stability for metabolites, and a lack of harmonization on minimum requirements for characterization. Standardization of correction factors and calculations could also lead to improved quality. The 
table provided in the ICH M10 BMV draft guideline offers guidance on the expected contents of a CoA [22]. It was confirmed that this information is helpful and adequate as a minimum requirement based on the challenges described above. Since any additional clarifications are difficult to add to a guideline, it was suggested that White Papers may be more suited to addressing the challenges industry is facing with reference standard providers. GCC is planning to fill this gap by writing this document from a global perspective.

\section{Recommendations}

Below is a summary of the discussions on the ICH M10 BMV draft guideline made during the $13^{\text {th }}$ WRIB expert panel and corroborated by the global bioanalytical community who actively participated in the three open forums.

\section{ICH M10 BMV draft guideline - chromatographic assays}

1. Hybrid LBA/LCMS recommendations need to be added to the ICH M10. The following fundamentals are a starting point that should be considered as minimum requirements:

- Specificity of the surrogate peptide(s);

- Digestion efficiency;

- Use of LBA (20\%/25\%) accuracy, precision and 30\% ISR criteria should be targeted until more experience with hybrid assays has accrued;

- Dilutional linearity;

- Capture reagent lot-to-lot variation.

2. Placement of the medium QC between 30-50\% of the ULOQ concentration on the arithmetic scale, especially for small-molecule assays in which the calibration curve range is wide;

3. An adequate number of QC levels should cover the full range of study samples, not just be placed anywhere within the profiles;

4. A trend in IS variability should be investigated. Sample internal standard responses should not be significantly different from the response for the calibration standards and QC samples;

5. Matrix effect: the accuracy and precision criteria should be clarified to apply to each of the individual lots;

6. Matrix factor provides important information on relative matrix effects, which could affect the visibility of the LLOQ. If an evaluation based solely on concentrations in spiked QCs is to be performed, peak areas should also be reported to show lot-to-lot variations on analyte responses.

\section{ICH M10 BMV draft guideline - LBA}

7. Parallelism should be used to investigate anomalous study data but should not be a routine validation assessment. The language of the ICH M10 draft guideline should be changed to require a justification of its applicability and an explanation of the results presented. Since it is an investigative tool for PK study samples, it is questioned if the $30 \%$ criterion is appropriate.

8. The use of single-well analysis is acceptable according to the ICH M10 guideline. It should be added in the ICH M10 BMV guideline that acceptance criteria are the same irrespective of the number of wells used per sample. As long as the method is validated using single-well analysis, then it is acceptable for use;

9. Although generally accepted as artificial as \% CV cannot be meaningfully determined with only two values; the use of two values to calculate $\% \mathrm{CV}$ between wells is the industry accepted procedure when using replicate analysis. It should be added in the ICH M10 BMV guideline that:

- A priori criteria should be defined in SOPs and validated, along with the specification of using either response values or concentration for the calculation;

- One well should not be deactivated to accept the sample. If the precision between wells does not meet the predefined criteria, the sample should be repeated.

10. The estimation of the impact of interference due to circulating targets should be assessed as well as whether total vs free assays should be measured using the molar ratio of target to drug based on what is expected in study. Note that post-dose target levels are usually much higher than baseline target levels; therefore, the post dose levels should be used to calculate target to drug molar ratios during validation:

- The interpretation of factors that impact LBA PK assays for measurements of biotherapeutics (e.g., concentrations of circulating target, assay reagents) should be evaluated based on what is expected with respect to drug mechanism of action; 
- When analyzing and reporting ADA interference in LBA PK assays, PD biomarkers can be a useful tool to assess impact of ADAs on target engagement when available.

11. Ultra-high concentration stability (see also Section 1 ):

- For LBA methods, the evaluation of high-concentration QC stability to account for the study sample concentration range does not need to be performed routinely. Such an assessment should be performed only as needed based on scientific rationale.

12. Stability at $-70^{\circ} \mathrm{C}$ : since no LBA data have been published that shows a protein is less stable at $-70^{\circ} \mathrm{C}$ than at $-20^{\circ} \mathrm{C}$, industry representatives suggested the requirement to perform stability evaluations at both temperatures should be removed.

\section{ICH M10 BMV draft guideline - common topics}

13. Whole blood stability should be performed using the plasma assay in validation:

- A method in whole blood should be used only during investigations if failure to meet stability criteria is suspected to be related to a partitioning effect rather than instability;

- The term "exploratory whole blood method" in the ICH M10 is misleading and should be removed or replaced with 'reliable whole blood method';

- The requirement to conduct this evaluation for a serum-based assay should be removed.

14. For lipemic testing, natural matrix is recommended in agreement with industry standards:

- Remove the sentence: ". . . it is acceptable to spike matrix with triglycerides even though it may not be representative of study samples";

- It should be added in the ICH M10 to use only certified hyperlipemic matrix with a CoA indicating at least $300 \mathrm{mg} / \mathrm{dl}$ triglyceride levels (lipemic index) or even higher if the study is in a special population with such high levels.

15. Robustness:

- For chromatographic assays, robustness is an operational issue already demonstrated by between-run $A \& P$ and ISR so no additional guidance is needed;

- For LBA, it was noted that robustness evaluations are often included in method development. Inclusion of formal robustness testing in method validation should be based on a risk analysis of the method taking into consideration the critical steps of the assay, the size and the length of the study and what had already been demonstrated during method development. If performed, a balanced design for robustness, testing all the extremes together, is recommended. If both extremes (e.g., longest vs shortest incubation times) meet criteria, then robustness is considered to have been demonstrated for all intermediate conditions.

16. CoA information in the ICH M10 is helpful and adequate as a minimum requirement;

17. It was clarified that when the M10 guidance states that stability evaluations must be compared to fresh calibrants and QCs, the intent is that the stability samples be assessed against the nominal concentrations.

\section{Minor issues and suggestions}

18. When using duplicate curves, the language is unclear regarding the option of dropping a calibrant level if necessary and still being able to obtain an acceptable regression. It should be possible to reject both replicates of a calibrant and still have a passing curve. Furthermore, it is inconsistent that a calibration curve level can be excluded when using a single curve, but not when a duplicate curve is used;

19. The ICH M10 BMV draft guideline does not discuss the evaluation of interference in the blank matrix sample or zero sample during study sample analysis. It is suggested to use the criteria from the EMA BMV LBA guideline [26], whereby interference above the LLOQ should be investigated;

20. The ICH M10 BMV draft guideline states that back-conversion of a metabolite should be evaluated when relevant. It should be clarified that all metabolites should be proven not to interfere with the assay;

21. It is proposed to use the EMA BMV wording "selectivity is tested by spiking at least 10 sources of sample matrix at or near the LLOQ" [26] for selectivity of LBA assays and remove the requirement for evaluating the high-QC level;

22. The dilutional linearity evaluation is recommended to be evaluated using at least three runs. It is suggested that this evaluation be performed using one run, as for other validation evaluations; 
23. Critical reagents: The section on minor/major changes should be simplified since the distinction between minor and major changes is not always straightforward and further investigations are needed due to the impact of these changes on sensitivity and specificity of the LBA. It is suggested to use the wording of the EMA guideline for critical reagents [26].

24. Regarding the overall evaluation of the intra- and inter-assay precision, consideration should be given to adding language allowing for a statistical analysis.

25. No criteria are presented for cross-validation. It is suggested to use similar wording as the EMA BMV guideline [26]: "For QC samples, the obtained mean accuracy by the different methods should be within 15\% [or $20 \%$ for LBA] and may be wider, if justified. For study samples, the difference between the two values obtained should be within 20\% [or 30\% for LBA] of the mean for at least 67\% of the repeats." Furthermore, this section states that cross-validation should be performed prior to sample analysis, however it also requires the use of incurred samples. It is suggested that the language be clarified.

26. The ICH M10 BMV draft guideline allows the use of stability data from another facility to support the assay. It is highly recommended that this sentence be removed completely or replaced with 'the sharing of stability data within different sites of the same company';

27. Regarding ISR values that are significantly different from the original result, it is suggested to add language stating that investigations should be considered in these cases.

\section{Section 3 - Input from regulatory agencies on bioanalysis \& BMV}

Brian Booth 1 , Seongeun (Julia) Cho ${ }^{1}$, Arindam Dasgupta ${ }^{1}$, Anna Edmison ${ }^{25}$, Sam Haidar ${ }^{1}$, John Kadavil' Sean Kassim ', Olivier Le Blaye ${ }^{22}$, Thais Correa Rocha ${ }^{23}$, Gustavo Mendes Lima Santos ${ }^{23}$, Catherine Soo ${ }^{25}$, Sriram Subramaniam ${ }^{1}$, Nilufer Tampal ${ }^{1}$, Theingi Thway', Stephen Vinter ${ }^{26}$, Jan Welink ${ }^{27}$, Emma Whale ${ }^{26}$ Authors are presented in alphabetical order of their last name. Author affiliations can be found at the beginning of the article.

\section{Regulatory input}

US FDA, EU EMA, Health Canada, UK MHRA, Brazil ANVISA, and France ANSM shared their views on regulated bioanalysis and BMV for the global bioanalytical community attending this event, to provide clarification on unresolved issues or expectations.

Bioresearch monitoring (BIMO) at the US FDA has a long tradition of ensuring the clinical and non-clinical research conducted in support of FDA regulated products supports the protection of human subjects, is adequate to support regulatory decisions, and is performed according to the regulations. The office of study Integrity and Surveillance (OSIS) in FDA/CDER/OTS strives to uphold this mission through BA/BE and GLP inspections and review. OSIS' creation 4 years ago was in large part to ensure the many domestic, and increasingly international, sites conducting clinical and bioanalytical BA/BE studies supporting drugs and therapeutic biologics were regularly evaluated in support of efficient and quality application review. The BIMO Compliance Program on In Vivo Bioequivalence [41] used by FDA employees during the inspections of BE studies was revised and divided into two new programs $[42,43]$. The first program includes specific instructions for the inspection of BA/BE clinical sites only, whereas the second program focuses on inspections of analytical sites. The analytical program draws from the FDA Inspection Operations Manual as well as the 2001 BMV guideline to provide inspectional directives for evaluated analytical site and site methodologies, but inspectors now refer to the 2018 BMV guidance [23] during inspections. Recent findings observed during inspections, focusing on the challenges seen in bioanalytical and analytical laboratories were shared. In fiscal year 2018, 1328 application inspections were performed or overseen domestically and internationally by the CDER BIMO program, which includes inspections by the Office of Scientific Investigations as well as OSIS inspections. OSIS' BA/BE program inspected and classified 241 site visits, 78 of which were focused on the analytical portion of BA/BE studies: $78 \%$ involved ANDAs, $11 \%$ covered NDAs, and $11 \%$ covered BLAs. The majority of the analytical site visits $(55 \%)$ resulted in no action indicated (NAI), $41 \%$ resulted in voluntary action indicated (VAI) and $4 \%$ in official action indicated (OAI). Inspectors focus on several critical points during bioanalytical inspections to ensure that studies supporting regulatory decisions are reliable. These critical points include pre-study method validation, run acceptance criteria, documentation of sample handling, repeat analysis, audit trails, equipment logs, SOPs, correspondence and comparison of onsite data with the study report. The top three deficiencies were related to stability (24\%), validation (10\%) and chromatography $(9 \%)$. 
The UK MHRA presented case studies demonstrating the importance of transparency in study reporting. When inspecting laboratories conducting bioanalysis, inspectors found that study reports do not always reflect the conduct of the study or the requirements of the protocol; key facts may be omitted or incorrect. Internal and external decisions are made using the data generated by these laboratories, and so reports are key for making decisions. When the reports are inaccurate and not representative of the data, there is a risk passed on to the decision makers that can affect regulatory approvals and patient/volunteer health. The responsibility of the reports rests with the data generators, and the transparency within internal systems is key to identifying issues before the reporting stage in order to eliminate significant problems later for the laboratory and public.

Health Canada presented its regulatory experience with large molecule review by the Clinical Evaluation Division - Hematology/Oncology. Health Canada reviewers follow the EMA BMV guideline [26] for the review of bioanalysis data and refer to the FDA immunogenicity guidance for ADA assays [44]. The first issue discussed was stability and dilutional linearity of samples above the ULOQ. Reviewers have observed cases where calibration ranges have not been extended, QCs have not been adapted or added, dilution QCs were not analyzed with study samples, there was lack of stability data available for the dilution QCs and samples were diluted beyond the MRD based on anticipated PK during original analysis. Sponsors have justified these cases due to LBAs limited dynamic range and the fact that dilutional linearity is performed during method validation. However, reviewers were concerned because dilutional linearity does not prove stability and all study samples should be originally analyzed using the same validated method to rule out bias. Health Canada recommends that, ideally, the calibration range should be extended as recommended in the EMA guideline and QC samples be adapted or added accordingly. If a new range is not possible, use the validated range for the original analysis of all study samples and then repeat those above the ULOQ following appropriate dilution and including dilution QCs. Ultra-high concentration stability should also be validated. The second issue presented was the reporting of bioanalytical repeats. Observations have included PK repeats or repeats due to anomalous concentrations may introduce bias in the study outcome; the reasons for repeats have been unclear and so reviewers cannot conclude that reassays were justified; criteria for accepting repeat values were not available; and there were failures when documenting the proportion of repeated samples and any investigations conducted. It was recommended that all repeated samples are identified and details are provided for each repeat; a list of codes is inadequate. Original and repeat values should be provided along with the criteria for reporting the final concentrations (an SOP is helpful). Finally, for reanalysis of pre-dose samples or those identified for a PK reason, it is very important that the reason for reanalysis be reported and justified, and that both the original and repeat concentrations be presented. Any details on investigations conducted to rule out analytical issues should be provided. When reviewing immunogenicity data, sponsors have contended that the long-term stability of positive controls were not relevant, but Health Canada reviewers were concerned by the lack of long-term stability data. The recommendation was to conduct long-term stability assessments, especially when samples are older than 24 months. During review of immunogenicity data, long-term stability data will be requested by reviewers. Freeze-thaw and short-term stability assessments and information on storage conditions of study samples should also be included. Another concern raised by reviewers is in regard to assay sensitivity and drug tolerance. Health Canada recommends that sampling for ADAs should be carried out at time points to minimize drug interference (e.g., at expected Ctrough).

The Brazil ANVISA presented its perspective on the ICH M10 BMV guideline. ANVISA plans to adopt the ICH M10 guideline as a resolution, as they do with their current BMV resolution [45]. However, this will have a regulatory impact on Brazilian BA/BE centers that will be required to implement new topics that have never been requested by ANVISA so far. These include cross-validations, whole blood stability, reinjection reproducibility, ISR, integration of chromatograms, recovery, LBA commercial and diagnostics kits and dried blood matrix. There are also some topics in their resolutions that will require some rewriting: scope (current scope is limited to BA/BE and will need to be expanded for ICH M10), reference standards, calibrations curves, selectivity, dilution integrity, and stock and working solutions. Other topics, including matrix effects, processed sample stability and endogenous compounds, will require changes in the way the assay is performed. Finally, documentation requirements outlined in ICH M10 BMV guideline will need to be addressed, either in the update of their current resolution [45] or as a revision of RDC 895/2003 [46].

\section{Q \& A}

Following the Regulatory Agencies' inputs, the floor was opened for an interactive discussion to all the regulators involved in Regulated Bioanalysis and BMV (US FDA, EU EMA, Health Canada, Brazil ANVISA, UK MHRA, 
France ANSM). The following includes the most important clarifications made and questions answered during the panel discussions:

- US FDA is currently inspecting using the 2018 FDA BMV guidance as a framework for expectations [23]. They will continue to do so until the ICH M10 BMV guideline is adopted in the US. When the ICH document is adopted, the FDA plans to use that guidance as the main BMV reference document;

- It was clarified that when the draft ICH M10 guideline states that stability evaluations must be compared to fresh calibrants and QCs, the intent is that the stability samples be assessed using fresh calibrants and QCs but compared to the nominal concentrations;

- If there is a significant QC outlier during sample analysis, regulators would like it included as well as removed in the inter-day precision and accuracy assessment. If the intra-day precision and accuracy are not acceptable for that run, this does not mean that regulators would consider that the run was not valid;

- Issues with cross-validations were discussed. Although industry understands the scientific rationale to perform cross-validation between laboratories, this can be difficult when multiple laboratories are used for different studies or when laboratories used earlier in the dossier no longer exist:

- Regulators were sympathetic to these issues and may allow some latitude depending on what the results demonstrate in the submission. Communication with the regulatory agency is highly encouraged;

- Cross-validation is not required when multiple clinical studies are not compared within a submission.

- Some regulators had the opinion that the analyte recovery does not need to be identical to the SIL-IS, but it should be consistent.

\section{Section 4 - Input from regulatory agencies on immunogenicity \& biomarkers}

Shashi Amur ${ }^{1}$, Abbas Bandukwala ${ }^{1}$, Elana Cherry ${ }^{25}$, Shirley Hopper ${ }^{28}$, Akiko Ishii-Watabe ${ }^{29}$, Susan Kirshner ${ }^{1}$, Kevin Maher ${ }^{1}$, Joao Pedras-Vasconcelos ${ }^{1}$, Yoshiro Saito ${ }^{29}$, Therese Solstad Saunders ${ }^{30}$, Venke Skibeli ${ }^{30}$, Daniela Verthelyi ${ }^{1}$, Yow-Ming Wang ${ }^{1}$, Haoheng Yan ${ }^{1}$

Authors are presented in alphabetical order of their last name. Author affiliations can be found at the beginning of the article.

Regulatory input

US FDA, EU EMA/Norway NoMA, Health Canada, UK MHRA, Japan MHLW, agencies shared their views on immunogenicity, biomarkers and gene therapy for the global bioanalytical community attending this event, to provide clarification on unresolved issues or expectations.

US FDA provided an overview of the finalized ADA assay validation guidance [44], focusing primarily on updates from the superseded $2016 \mathrm{draft}$ guidance. To facilitate both immunogenicity assessments during clinical development of therapeutic biologics and the subsequent regulatory review processes, the US FDA has published several immunogenicity guidances over the last 10 years culminating in the eagerly awaited and finalized ADA assay validation guidance published in January 2019.

- The guidance provides recommendations to facilitate development of immune assays, including screening, confirmatory, titering and neutralizing antibody assays for assessment of the immunogenicity of therapeutic proteins during clinical trials for 351(a) innovator and 351(k) biosimilar biologics regulated under the United States Public Health Service Act;

- Regarding neutralizing antibody assays, FDA recommends the use of cell-based bioassays as they tend to be more reflective of the in vivo situation. However, a competitive ligand-binding assay may be a feasible alternative in many situations. In selected cases, where there is a highly sensitive PD marker and the PK/PD assay is appropriately designed to inform changes to clinical activity over time, it may be possible to use these in lieu of a formal neutralizing antibody assay. FDA should be consulted when planning to use alternative approaches;

- When evaluating assay sensitivity, the serial dilutions should be no greater than two or threefold, and a minimum of five dilutions should be tested. During routine performance of the assay, a low PC system suitability control, consistently demonstrated as positive is used in both screening and confirmatory assays to ensure that the sensitivity of the assays is acceptable across assay runs.

- Drug tolerance should be tested by varying both the concentration of the PC and the amount of the drug;

- Samples for precision determination should include negative controls and positive samples whose testing yields low, intermediate, and high values of the assay dynamic range; 
- MRD typically ranges from 1:5 to 1:100. All sample dilutions, including the MRD and acid dissociation steps, should be factored into the calculations of titers and sensitivity;

- Data demonstrating suitable recovery for any sample pre-treatment steps such as acid dissociation should be provided in the validation report;

- The use of a living integrated immunogenicity summary document that sponsors begin populating early in product development and updated as clinical programs progress through IND stages into BLA and post-approval is recommended;

- Integrated summaries of immunogenicity are requested for:

- All new 351(a) and 351(k) BLA submissions; sponsors will receive an information request if an integrated summary of immunogenicity is absent at the time of BLA filing;

- New supplemental BLA efficacy supplements sponsors should demonstrate that the cut point determined during the original validation applies to the novel clinical population studied in the Efficacy supplement. If clinical ADA rates were not determined in the efficacy study, sponsors should include a suitable justification as part of immunogenicity risk assessment;

- New NDAs for oligonucleotide, peptide, or carbohydrate drugs are recommended to include integrated summaries of immunogenicity;

- New and ongoing INDs are suggested to include integrated summaries of immunogenicity with stage appropriate information. Information should be updated as clinical program progresses.

US FDA also provided a regulatory perspective on cut point calculation approaches and statistical methods, discussing FDA's risk-based evaluation on cut point determination approaches and updates on cut points from their 2019 ADA guidance [44]. Cut points are one of the essential parameters the validation of in immunogenicity assays. They can be influenced by a myriad of interfering products and matrix components in the study samples. Factors such as pre-existing antibodies in the study population further complicate the task of determining appropriate cut points. The FDA recommends cut points be statistically determined using treatment-naïve ADA negative samples. Study design and statistical methods used for cut point determination vary among applications. Commercial sourced samples from normal and/or patient populations are commonly used for cut point determination during initial assay validation. The suitability of these cut points determined during validation should be confirmed in-study using treatment-naïve samples from the clinical study.

Several types of cut point are used in immunogenicity assays including floating cut point, fixed cut point and dynamic cut point. Floating cut points apply a correction factor to the negative control in each run and are the most common approach for screening assays. Fixed cut points are most commonly seen for confirmatory and neutralizing assays. Dynamic cut points can change between plates in a run, between runs in a study, or between studies, and do not depend on pre-study validation. The use of dynamic cut points may indicate that the assay requires further optimization. The use of dynamic cut points is not recommended.

The screening assay cut point should provide a 5\% false-positive rate (FPR) to reduce the risk of false-negative results. Using the lower $90 \%$ confidence limit (LCL) of the 95th percentile helps ensure a 5\% FPR 90\% of the time. Particularly for high-risk products (with potential clinical impact of ADA) the more conservative $90 \%$ lower confidence limit cut point should be considered. Other approaches may be used (point estimate, median + median absolute deviation), but should be justified.

Because clinical population, sample collection, and sample storage can impact assay background signal, the cut point determined during validation should be verified on pre-treatment study samples. If the assay signals from pre-treatment samples differ significantly from the validation samples, an in-study cut point should be established to obtain a $5 \%$ FPR in pre-treatment samples for screening assay, and subsequently a $1 \%$ FPR for confirmatory assay. Samples with pre-existing ADA should be excluded from the cut point determination but should be monitored for boosted response after exposure.

The most frequently used method for setting up the cut point for confirmatory assays is to spike drug-naïve samples with drug and compare the signal from spiked vs unspiked samples. The mean and standard deviation of the $\%$ change is then used to calculate the cut point based on a $1 \%$ false-positive rate.

Titer is determined from the reciprocal of the highest dilution that gives a value at or just above the cut point of the assay or by extrapolating the dilution to the assay cut point using the linear portion of the dose response curve. The titer cut point may be the same as the screening cut point or statistically determined based on $1 \%$ or $0.1 \%$ FPR. 
Health Canada discussed unwanted immunogenicity, an immune response by an organism against a therapeutic antigen that has the potential to inactivate the therapeutic effects of the treatment and, in some cases, induce adverse effects. Given the potential risks associated with unwanted immunogenicity, drug submissions submitted to Health Canada should include an adequate characterization of the immunogenicity profile of a biologic drug to enable regulators to assess the clinical consequences of product immunogenicity. Regulatory decisions are made on a case-by-case basis depending on numerous considerations that affect the benefit/risk profile of a drug. Regulators also rely on the overarching life-cycle approach to mitigate risks associated with immunogenicity.

EU EMA/Norway NoMA presented the agency's regulatory perspective on clinical relevance of ADAs. There are guidance documents available from the EMA for immunogenicity, one related to therapeutic proteins [47] and the other to monoclonal antibodies [48]. There are patient-related factors and product-related factors that may influence the development of an immune response. Patient-related factors include genetics, age and pre-existing reactivity. Product-related factors include protein structure and post-translational modifications, impurities and aggregation. Intermittent administration of a biotherapeutic can also give a higher incidence of ADA-production. The consequences of these immune responses can be transient or persistent ADAs, which can affect PK/PD, efficacy and safety of the product. Therefore, it is important to investigate the relationship between dose, exposure, efficacy and toxicity of the therapeutic protein.

For low-risk biotherapeutics, frequent sampling is typical at the beginning, however, samples are normally only assayed at the end of the trial and at baseline. Shorter follow up is usually required with routine pharmacovigilence for immunogenicity planned. For high-risk biotherapeutics, more frequent sampling may be needed and samples could potentially be assayed in real time (typically with a cell-based neutralization assay) in order to increase clinical monitoring. Follow-ups are generally longer, and post-marketing immunogenicity studies may be required. There are various approaches when designing the sampling plan for ADAs. Sampling can be based on the PK profile, risk based to support the immunogenicity program or symptom driven if suspicion of an unwanted immune response. Testing of immunogenicity should be designed to discern transient from persistent ADAs. The EU guideline recommends 1 year of immunogenicity data in case of chronic treatment. ADAs may sustain circulation and/or enhance clearance of therapeutic proteins which may have clinical relevance by impacting efficacy and exposure. When evaluating the impact on safety and efficacy, it is important to address the maturation of an emerging immune response by determining if the ADAs are neutralizing, and if further characterization is needed, also isotyping, T-cell involvement, or if a threshold level of ADAs can be determined. It may be possible to manage immunogenicity by co-medication or with a mitigation plan to the presciber. When investigating immunogenicity of a biosimilar, regulators are looking to see if there is increased or decreased response compared to the reference product. Relative/comparable immunogenicity of biosimilars needs to be demonstrated pre-licensing. Planning and assessment of emerging immunogenicity requires multidisciplinary team work using a risk-based approach, and immunogenicity assessments need to be integrated into PK/PD, safety and efficacy to look for correlations to clinical consequences. An adequate integrated summary of the immunogenicity program can significantly benefit regulatory assessments.

US FDA presented a clinical pharmacology perspective on the use of biomarker data in support of regulatory submissions. The context of use is important to consider for PD biomarkers. The intended purpose of PD biomarkers evolves through the different phases of new drug development programs. In early stages of clinical development, biomarkers can provide information on extent of target engagement, proof of biology and confirmation of therapeutic MOA and enable modeling of PK-PD to identify efficacious dose levels for later stage development. The utility of PD biomarkers in biosimilar drug development is to assess clinically meaningful differences between the proposed biosimilar and the reference product.

Biomarkers can support a demonstration of biosimilarity by reducing the residual uncertainty regarding the existence of any clinically meaningful differences between products. There are five characteristics to consider when selecting PD biomarkers:

1. The anticipated time of onset of change in the PD biomarker relative to dosing and its return to baseline with discontinuation of dosing;

2. The dynamic range of the PD biomarker over the exposure range to the biological product;

3. The sensitivity of the PD biomarker to differences between the proposed biosimilar product and the reference product;

4. The relevance of the PD biomarker to the MOA of the drug; 
5. The analytical validity of the PD biomarker assay.

Bioanalytical method validation is critical for PD biomarkers intended for quantitative assessments and support of regulatory action.

In partnership with test manufacturers and clinical scientists, US FDA reviews data that demonstrate the safety and effectiveness of devices, which, for in vitro diagnostic tests, is typically demonstrated through analytical and clinical performance testing. US FDA provided an overview of the premarket clearance/approval process with discussion of validation studies that are typically seen for flow cytometry tests. Tests are evaluated based on their intended use/indications for use (IU/IFU). Intended use refers to the general purpose of the device or its function and encompasses the indications for use. The indications for use, as defined in 21 CFR 814.20(b)(3)(i), describes the disease or condition the device will diagnose, treat, prevent, cure or mitigate, including a description of the patient population for which the device is intended [49]. For in vitro diagnostic tests, the IU/IFU typically outlines the analytical parameters that will be validated and reported, the instrumentation required, the clinical purpose, the target condition, the target population and the setting (e.g., clinical laboratory, point-of care). Validation of a flow cytometric device typically includes demonstrating that precision, reproducibility, sensitivity, specificity, linearity, carryover, reference range and accuracy are adequate and ensure that the IVD can be used in a safe and effective manner, when used according to the IU/IFU. Device classification and availability of similar IVD's that can serve as comparators of performance also affect the regulatory path for premarket clearance and approval. US FDA also discussed resources available from CLSI that provide more information on use and validation [50-56].

Flow cytometry is a powerful analytical tool that has been widely used to perform immunophenotyping, cell counting and assessing numerous cell functions such as intracellular cytokine production, protein phosphorylation, cell proliferation, apoptosis and so on. These functions make flow cytometry a widely-used tool in establishing identity, purity, and potency of cell and gene therapy products. US FDA presented the concept and strategies for flow cytometry standardization and the measures to be taken to ensure consistency in product characterization and manufacturing of high quality, safe and effective gene cell therapy products. One of the critical challenges in flow cytometry is reducing inter-laboratory and intra-laboratory (between runs, between days, and instrument to instrument) variabilities to ensure consistent and reproducible results. FDA recommended performing routine instrument calibration (QC/QA) and establishing additional strategies to reduce these variabilities. These additional strategies could be achieved by optimizing the PMT voltage for each detector to ensure that negative events are clearly distinguishable from electronic noise, dim positive events can be discriminated from the negative, and the high positive populations are within the linear range of each detector. It is recommended that the PMT voltage optimization includes both unstained and stained cells in the standardization strategy. Unstained cells help to optimize the PMT voltages to ensure that the generated signals are discriminated from instrument electronic noise (e.g., using the $2.5 \mathrm{rSDEN}$ approach). Next, stained cells can be used to verify that the brightest populations are within the linear scale for each detector.

When the optimal PMT voltages are determined, it is recommended to save these settings and record the target MFI values using fluorescently labeled beads. Clinical samples and cell controls should be run using the same optimized settings. Identifying target values is crucial to monitor instrument performance over time. These values are also important to achieve multi-instrument standardization by adjusting the PMT voltage of the other cytometers using the same fluorescently labeled beads. After multi-instrument standardization, multicolor stained samples should be run to ensure comparable results.

Standardization strategy can be affected by properties of clinical samples and assay reagents. FDA recommended to consider the following factors when performing standardization: the clinical sample (e.g., cell autofluorescence, sample preparation, antigen density) and the assay reagent (e.g., antibody clone, fluorochrome conjugate, antibody fluorochrome to protein ratio).

UK MHRA presented its perspective on predictive biomarker (BM)-based assays. Companion diagnostics (CDx) are a subset of predictive BM-based assays and are defined for the first time in the new European in vitro diagnostic regulation (IVDR) [57]. Under IVDR classification rules for CDx, the demonstration of analytical performance and clinical performance is required. A new EMA guideline on 'predictive biomarker-based assay development in the context of drug development and lifecycle' is under development [58]. The aim is to optimize co-development of personalized medicines and CDx. Data from clinical drug trials may provide evidence of the clinical performance of a co-developed CDx. Therefore, it is important to ensure that BM assays used in clinical drug trials are adequately validated for the intended purpose. To minimize the risk of an incorrect result and protect 
study subjects, the level of validation should be particularly stringent if the BM test is used to enroll patients or decide treatment. The derivation of clinical performance characteristics such as diagnostic sensitivity and specificity depends on the inclusion of both BM-positive and BM-negative patients within the same drug trial. However, it is sometimes established early on that drug response is determined by the presence or absence of a predictive BM. Later clinical trials are likely to be of enriched design such that BM-negative (or BM-positive) patients are excluded, and it will not be possible to formally validate the predictive BM during the pivotal drug trial. Instead, the study will provide evidence of drug response in the population selected by the CDx. This approach may be adequate for demonstration of clinical performance if fully justified. If the $\mathrm{CDx}$ is used to assign $\mathrm{BM}$ status for the pivotal trial, then the trial data provide direct demonstration of clinical performance. If the clinical trial assay is not the fully developed $\mathrm{CDx}$, an additional bridging study using clinical samples may be required.

US FDA provided case studies on recent biomarker qualifications and analytical validation. The specific context of use for a biomarker drives the extent of evidence needed for qualification. Analytical validation establishes performance characteristics of assays used to measure or assess the biomarker and can include parameters such as accuracy, reference ranges, and precision. Analytical validation is not the same as clinical validation, which establishes that the biomarker acceptability identifies, measures or predicts the concept of interest.

For the development of biomarkers, Japan MHLW presented that analytical validation in addition to clinical usefulness is required. However, unlike exogenous drugs, biomarker assay validation can be more challenging. For example, the biomarker analyte is endogenous to the matrix and the structure of the recombinant protein as a standard is often different from the endogenous analyte. Due to these issues, application of bioanalytical method validation guidelines for PK for biomarkers is typically not appropriate. The US Critical Path Institute (C-Path) draft White Paper [28] and the FDA BMV guidance [23] include biomarkers in their scope to provide guidance for bioanalysts developing and validating biomarkers. In Japan, the AMED research group consisting of regulatory and industry members created a survey for pharmaceutical companies in Japan to understand the current situation on biomarker assay validation for clinical trials [59]. Referring to its results, the AMED research group has started to draft a 'Points to Consider' document on BAV in Japan. There have been four meetings so far, focusing on discussions regarding scope, matrix, standards, selectivity, specificity, calibration curve, matrix effect, accuracy, precision, parallelism, dilution linearity, stability and ISR. The scope of the document will be very close to the C-Path document and instructive not only for bioanalysts but for toxicology and pharmacology researchers as well.

The US FDA presented CDER's regulatory science initiatives. US FDA defines regulatory science as the science of developing new tools, standards, and approaches to assess the safety, effectiveness, quality, toxicity, public health impact, or performance of FDA regulated products. Advancing regulatory science to better inform regulatory decision making and protecting and promoting public health is fundamental to FDA's core mission. CDER is one of the centers at FDA and its regulatory science activities are aimed at expediting the availability of new drugs while ensuring that they are safe and effective. CDER's approach is threefold: 1) create new methods, tools, and analytical approaches that can support and accelerate the evaluation of new drug products; 2) develop new processes and technologies that help ensure that drug products are manufactured to the highest quality standards; and 3) build information systems and computational tools that can help predict and promptly identify issues with the safety and effectiveness of marketed drugs. CDER is engaged in regulatory science-relevant research activities and collaborates with other centers in FDA and with stakeholders outside FDA. Internal and external funding mechanisms to support research in regulatory science are available at CDER and scientific achievement awards are given to researchers to recognize outstanding work. Information on these research projects is captured in the CDER Science Database. The number of projects tracked in the CDER database for the years 2015-2017 are: 333 for 2015; 386 for 2016 and 462 for 2017.

\section{$Q$ \& $A$}

Following the regulatory agencies' input, the floor was opened for an interactive discussion to all the regulators involved in immunogenicity, biomarkers and gene therapy (US FDA, EU EMA/NoMA, Health Canada, Japan MHLW, UK MHRA). The following includes the most important clarifications that were made and questions answered during the panel discussions:

- The expectation for long-term stability for ADA assays is that literature reports that samples are typically stable for 2 years. If samples are stored more than 2 years, a risk assessment is required regarding the need to perform long-term stability; 
- If alternative approaches are being considered for the determination of cut points, sponsors are encouraged to contact regulators to discuss and obtain feedback. Justification of the approach is important;

- The same cut point can be used for different studies and indications as long as the cut point is verified in the treatment-naïve samples from the studies for indications. The verification process should include excluding outliers in the sample set followed by assessing the screening false-positive rate using the determined cut point. It is not necessary to reassess the cut point every time, but a statistical justification should be provided if cut point is not reassessed;

- Expect to present relevant CMC information in the integrated summary of immunogenicity and/or CTD module 2 S2.6 (Manufacturing Process Development). Typically, regulators are seeing 60-100+ pages, the higher end mostly with legacy products;

- If a NAb assay is not available when the BLA is submitted, it is unlikely to be a filing issue, but whether it is an approvability issue will depend on the immunogenicity risk and clinical data. The inclusion of a NAb assay in the original BLA submission should be discussed with health authorities prior to BLA submission;

- Because the 2018 FDA BMV guidance includes ISR for BAV, Japan currently tentatively proposes to include a similar recommendation;

- There is industry/regulator agreement that there is a need for a future international harmonized guidance on BAV.

\section{Acknowledgments}

- The US FDA, Europe EMA, UK MHRA, Brazil ANVISA, Health Canada, Japan MHLW, France ANSM and Norway NoMA for supporting this workshop.

- B Booth (US FDA), C Fandozzi (Merck), C Evans (GlaxoSmthKline), R Pillutla (Bristol-Myers Squibb), S Kaur (Genetech), H Yu (Boeringer Ingelheim), F Garofolo (Angelini Pharma), B Schweighardt (BioMarin), M Liang (AstraZeneca), L Stevenson (Biogen); M Buonarati (Inertek), A Vitaliti (Novartis), V Litwin (Caprion), D Mehta (Biogen), S Piccoli (GlaxoSmithKline), B Gorovitz (Pfizer), R Palmer (Sanofi), L Amavaradi (Shire/Takeda), C Beaver (Syneos), S Richards (Sanofi), M Rajadhyaksha (Regeneron) for chairing the workshop and/or the White Paper discussions.

- All the workshop attendees and members of the global bioanalytical community who have sent comments and suggestions to the workshop to complete this White Paper.

- Future Science Group is a trusted partner.

Financial \& competing interests disclosure

The authors have no relevant affiliations or financial involvement with any organization or entity with a financial interest in or financial conflict with the subject matter or materials discussed in the manuscript. This includes employment, consultancies, honoraria, stock ownership or options, expert testimony, grants or patents received or pending, or royalties.

No writing assistance was utilized in the production of this manuscript.

\section{References}

1. Savoie N, Booth BP, Bradley T et al. 2008 White Paper: The 2nd Calibration and Validation Group Workshop on Recent Issues in Good Laboratory Practice Bioanalysis. Bioanalysis 1(1), 19-30 (2009).

2. Savoie N, Garofolo F, van Amsterdam P et al. 2009 White Paper on Recent Issues in Regulated Bioanalysis from the 3rd Calibration and Validation Group Workshop. Bioanalysis 2(1), 53-68 (2010).

3. Savoie N, Garofolo F, van Amsterdam P et al. 2010 White Paper on Recent Issues in Regulated Bioanalysis and Global Harmonization of Bioanalytical Guidance. Bioanalysis 2(12), 1945-1960 (2010).

4. Garofolo F, Rocci M, Dumont I et al. 2011 White Paper on Recent Issues in Bioanalysis and Regulatory Findings from Audits and Inspections. Bioanalysis 3(18), 2081-2096 (2011).

5. DeSilva B, Garofolo F, Rocci M et al. 2012 White Paper on Recent Issues in Bioanalysis and Alignment of Multiple Guidelines. Bioanalysis 4(18), 2213-2226 (2012).

6. Stevenson L, Rocci M, Garofolo F et al. 2013 White Paper on Recent Issues in Bioanalysis: "Hybrid” - the best of LBA \& LC/MS. Bioanalysis 5(23), 2903-2918 (2013).

7. Fluhler E, Hayes R, Garofolo F et al. 2014 White Paper on recent issues in bioanalysis: a full immersion in bioanalysis (Part 1 - small molecules by LCMS). Bioanalysis 6(22), 3039-3049 (2014).

8. Dufield D, Neubert H, Garofolo F et al. 2014 White Paper on recent issues in bioanalysis: a full immersion in bioanalysis (Part 2 hybrid LBA/LCMS, ELN \& regulatory agencies' input). Bioanalysis 6(23), 3237-3249 (2014). 
9. Stevenson L, Amaravadi L, Myler H et al. 2014 White Paper on recent issues in bioanalysis: a full immersion in bioanalysis (Part 3 - LBA and immunogenicity). Bioanalysis 6(24), 3355-3368 (2014).

10. Welink J, Fluhler E, Hughes N et al. 2015 White Paper on recent issues in bioanalysis: focus on new technologies and biomarkers (Part 1 - small molecules by LCMS). Bioanalysis 7(22), 2913-2925 (2015).

11. Ackermann B, Neubert H, Hughes $\mathrm{N}$ et al. 2015 White Paper on recent issues in bioanalysis: focus on new technologies and biomarkers (Part 2 - hybrid LBA/LCMS and input from regulatory agencies). Bioanalysis 7(23), 3019-3034 (2015).

12. Amaravadi L, Song A, Myler H et al. 2015 White Paper on recent issues in bioanalysis: focus on new technologies and biomarkers (Part 3 - LBA, biomarkers and immunogenicity). Bioanalysis 7(24), 3107-3124 (2015).

13. Yang E, Welink J, Cape S et al. 2016 White Paper on recent issues in bioanalysis: focus on biomarker assay validation (BAV) (Part 1 small molecules, peptides and small molecule biomarkers by LCMS). Bioanalysis 8(22), 2363-2378 (2016).

14. Song A, Lee A, Garofolo F et al. 2016 White Paper on recent issues in bioanalysis: focus on biomarker assay validation (BAV): (Part 2 Hybrid LBA/LCMS and input from regulatory agencies). Bioanalysis 8(23), 2457-2474 (2016).

15. Richards S, Amaravadi L, Pillutla R et al. 2016 White Paper on recent issues in bioanalysis: focus on biomarker assay validation (BAV): (Part 3 - LBA, biomarkers and immunogenicity). Bioanalysis 8(23), 2475-2496 (2016).

16. Welink J, Yang E, Hughes N et al. 2017 White Paper on recent issues in bioanalysis: aren't BMV guidance/guidelines 'Scientific'? (Part 1 - LCMS: small molecules, peptides and small molecule biomarkers). Bioanalysis 9(22), 1807-1825 (2017).

17. Neubert H, Song A, Lee A et al. 2017 White Paper on recent issues in bioanalysis: rise of hybrid LBA/LCMS immunogenicity assays (Part 2: hybrid LBA/LCMS biotherapeutics, biomarkers \& immunogenicity assays and regulatory agencies' inputs). Bioanalysis 9(23), 1895-1912 (2017).

18. Gupta S, Richards S, Amaravadi L et al. 2017 White Paper on recent issues in bioanalysis: a global perspective on immunogenicity guidelines \& biomarker assay performance (Part 3 - LBA: immunogenicity, biomarkers and PK assays). Bioanalysis 9(24), 1967-1996 (2017)

19. Welink J, Xu Y, Yang E et al. 2018 White Paper on Recent Issues in Bioanalysis: 'A global bioanalytical community perspective on last decade of incurred samples reanalysis (ISR)' (Part 1 - small molecule regulated bioanalysis, small molecule biomarkers, peptides \& oligonucleotide bioanalysis). Bioanalysis 10(22), (1781-1801 (2018).

20. Neubert H, Olah T, Lee A et al. 2018 White Paper on Recent Issues in Bioanalysis: focus on immunogenicity assays by hybrid LBA/LCMS and regulatory feedback (Part $2-\mathrm{PK}, \mathrm{PD} \& \mathrm{ADA}$ assays by hybrid LBA/LCMS \& regulatory agencies' inputs on bioanalysis, biomarkers and immunogenicity). Bioanalysis 10(23), 1897-1917 (2018).

21. Stevenson L, Richards S, Pillutla R et al. 2018 White Paper on Recent Issues in Bioanalysis: focus on flow cytometry, gene therapy, cut points and key clarifications on BAV (Part 3 - LBA/cell-based assays: immunogenicity, biomarkers and PK assays). Bioanalysis 10(24), 1973-2001 (2018).

22. ICH M10, Draft Bioanalytical Method Validation (2019). https://www.ich.org/fileadmin/Public_Web_Site/ICH_Products/Guidelines /Multidisciplinary/M10/M10EWG_Step2_DraftGuideline_2019_0226.pdf

23. US Department of Health and Human Services, Guidance for Industry, Bioanalytical Method Validation. US FDA, Center for Drug Evaluation and Research, Center for Veterinary Medicine, MD, USA (2018).

24. US Department of Health and Human Services, Draft Guidance for Industry, Bioanalytical Method Validation. US FDA, Center for Drug Evaluation and Research, Center for Veterinary Medicine, MD, USA (2013).

25. US Department of Health and Human Services, Guidance for Industry, Bioanalytical Method Validation. US FDA, Center for Drug Evaluation and Research, Center for Veterinary Medicine, MD, USA (2001)

26. European Medicines Agency. Guideline on Bioanalytical Method Validation. Committee for Medicinal Products for Human Use (CHMP), London, UK (2011).

27. Lee JW, Devanarayan V, Barrett YC et al. Fit-for-purpose method development and validation for successful biomarker measurement. Pharm. Res. 23(2), 312-328 (2006).

28. Piccoli SP, Sauer JM. Points to consider document: scientific and regulatory considerations for the analytical validation of assays used in the qualification of biomarkers in biological matrices biomarker assay collaborative evidentiary considerations writing group, critical path institute (c-path) (2017)

https://healthpolicy.duke.edu/sites/default/files/atoms/files/cpath_ptc_biomarker_qualification_assays_20170623.pdf

29. Garofolo W, Savoie N. The decennial index of the white papers in bioanalysis: 'a decade of recommendations (2007-2016)'. Bioanalysis $9(21), 1681-1704$ (2017).

30. Lowes $\mathrm{S}$, Boterman M, Doig M et al. Recommendations on bioanalytical method stability implications of co-administered and co-formulated drugs by Global CRO Council for Bioanalysis (GCC). Bioanalysis 4(17), 2117-2126 (2012).

31. Kelley M, Beaver C, Stevenson L et al. Large molecule run acceptance: recommendation for best practices and harmonization from the global bioanalysis consortium harmonization team. AAPS J. 16(2), 221-225 (2014).

32. ICH M10, Final endorsed concept paper M10: bioanalytical method validation (2016). https://www.ich.org/fileadmin/Public_Web_Sit e/ICH_Products/Guidelines/Multidisciplinary/M10/ICH_M10_Concept_paper_final_7Oct2016.pdf 
33. Lowes S, Jersey J, Shoup R et al. Recommendations on: internal standard criteria, stability, incurred sample reanalysis and recent 483 s by the Global CRO Council for Bioanalysis. Bioanalysis 3(12), 1323-32 (2011).

34. Bower J, Fast D, Garofolo F et al. 8th GCC: consolidated feedback to US FDA on the 2013 Draft FDA guidance on bioanalytical method validation. Bioanalysis 6(22), 2957-2963 (2014).

35. Hayes R, LeLacheur R, Dumont I et al. 9th GCC closed forum: CAPA in regulated bioanalysis; method robustness, biosimilars, preclinical method validation, endogenous biomarkers, whole blood stability, regulatory audit experiences and electronic laboratory notebooks. Bioanalysis 8(6), 487-495 (2016).

36. Briscoe C, Hughes N, Hayes R et al. 12th GCC Closed forum: critical reagents; oligonucleotides; CoA; method transfer; HRMS; flow cytometry; regulatory findings; stability; and immunogenicity. Bioanalysis 11(12), 1129-1138 (2019).

37. Committee for medicinal products for human use (CHMP) overview of comments received on 'guideline on validation of bioanalytical methods' (2011). https://www.ema.europa.eu/en/documents/other/overview-comments-received-guideline-validation-bioanalytical-met hods-emea/chmp/ewp/192217/2009_en.pdf

38. Stevenson L, Purushothama S. Parallelism: considerations for the development, validation and implementation of PK and biomarker ligand-binding assays. Bioanalysis 6(2), 185-198 (2014).

39. Rocci M, Lowes S, Shoup R et al. 7th GCC Insights: incurred samples use; fit-for-purpose validation, solution stability, electronic laboratory notebook and hyperlipidemic matrix testing. Bioanalysis 6(20), 2713- 2720 (2014).

40. ICH Q2(R1), Validation of analytical procedures: text and methodology (1994). https://www.ich.org/fileadmin/Public_Web_Site/ICH_Products/Guidelines/Quality/Q2_R1/Step4/Q2_R1__Guideline.pdf

41. FDA compliance program chapter 48 - bioresearch monitoring human drugs in vivo bioequivalence (7348.001) (1999). https://www.fda.gov/media/75916/download

42. FDA Compliance Program Chapter 48 - Bioresearch Monitoring. Procedure for FDA staff: in vivo bioavailability/bioequivalence studies (clinical) (7348.003) (2018). https://www.fda.gov/media/112538/download

43. FDA Compliance Program Chapter 48 - Bioresearch Monitoring. Procedure for FDA staff: in vivo bioavailability/bioequivalence studies (analytical) (7348.004) (2018). https://www.fda.gov/media/112533/download

44. US Department of Health and Human Services. Guidance For Industry, Immunogenicity Testing of Therapeutic Protein Products Developing and Validating Assays for Anti-Drug Antibody Detection. US FDA, Center for Drug Evaluation and Research, Center for Biologics Evaluation and Research, MD, USA (2019).

45. ANVISA. Resolution RDC 27 Minimum Requirements for Bioanalytical Method Validation Used in Studies with the Purpose of Registration and Post-Registration of Medicines. Agencia National de Vigilancia Sanitaria, Brazil (2012).

46. European Commission. Guidelines for Elaborating a Relative Bioavailability/Bioequivalence Study's Technical Report. Resolution 895. ANVISA, Brazil (2003).

47. European Medicines Agency. Guideline on Immunogenicity Assessment of Therapeutic Proteins. Committee for Medicinal Products for Human Use (CHMP). London, UK (2017).

48. European Medicines Agency. Immunogenicity Assessment of Monoclonal Antibodies Intended for in vivo Clinical Use. Committee for Medicinal Products for Human Use (CHMP). London, UK (2012).

49. US Department of Health and Human Services, Guidance for Industry, the 510(k) Program: Evaluating Substantial Equivalence in Premarket Notifications [510(k)]. US FDA, Center for Devices and Radiological Health, Center for Biologics Evaluation and Research. MD, USA (2014).

50. CLSI Guidance EP05-A3 Evaluation of Precision of Quantitative Measurement Procedures (2014).

51. CLSI Guidance EP06-A Evaluation of the Linearity of Quantitative Measurement Procedures: A Statistical Approach (2003).

52. CLSI Guidance. EP07Interference Testing in Clinical Chemistry (2018).

53. CLSI Guidance EP12-A2 User Protocol for Evaluation of Qualitative Test Performance (2008).

54. CLSI Guidance EP17-A2: Evaluation of Detection Capability for Clinical Laboratory Measurement Procedures (2012).

55. CLSI Guidance EP24-A2 Assessment of the Diagnostic Accuracy of Laboratory Tests Using Receiver Operating Characteristic Curves (2011).

56. CLSI Guidance EP28-A3C Defining, Establishing and Verifying Reference Intervals in the Clinical Laboratory (2010).

57. European Commission In Vitro Diagnostic Regulation (IVDR 2017/746).

58. European Medicines Agency. Concept paper on predictive biomarker-based assay development in the context of drug development and lifecycle. Committee for Medicinal Products for Human Use (CHMP), London, UK (2017).

59. Ohtsu Y, Matsumaru T, Katashima M et al. Biomarker assay validation for clinical trials: a questionnaire survey to pharmaceutical companies in Japan. Bioanalysis 11(2), 55-60 (2018). 
60. FDA-NIH Biomarker Working Group. BEST (Biomarkers, EndpointS, and other Tools) Resource. BEST (Biomarkers, EndpointS, and other Tools) Resource. Silver Spring (MD): Food and Drug Administration (US); Bethesda (MD): National Institutes of Health (US); (2016).

61. Mayrand-Provencher L, Furtado M, Mess J-N et al. Choosing the appropriate matrix to perform a scientifically meaningful lipemic plasma test in bioanalytical method validation. Bioanalysis 6(12), 1639-1646 (2014).

62. Stevenson L, Kelley M, Gorovits B et al. Large molecule specific assay operation: recommendation for best practices and harmonization from the global bioanalysis consortium harmonization team. AAPS J. 16(1), 83-88 (2014).

63. Hougton R, Gouty D, Allinson J et al. Recommendations on biomarker bioanalytical method validation by GCC. Bioanalysis 4(20), 2439-2446 (2012). 